\title{
Facilitators and barriers to child restraint use in motor vehicles: a qualitative evidence synthesis
}

Soumyadeep Bhaumik1, Kate Hunter2,5, Richard Matzopoulos 3,4, Megan Prinsloo4, Rebecca Ivers 5 , Margaret Peden 5,6

1. The George Institute for Global Health India , New Delhi, India

2. The George Institute for Global Health Australia, Sydney, Australia

3. School of Public Health \& Family Medicine, University of Cape Town, Cape Town, South Africa

4. Burden of Disease Research Unit, South African Medical Research Council, Tygerberg, South Africa

5. School of Public Health and Community Medicine, University of New South Wales, Sydney, Australia

6. The George Institute for Global Health UK, Oxford University, Oxford, United Kingdom

Abstract-431 words

\section{Abstract}

\section{Background}

Road traffic collisions contribute a significant burden of mortality and morbidity to children globally. The improper or non-use of child restraints can result in children sustaining significant injuries in the event of a collision. Systematic reviews on the effectiveness of various interventions to increase the use of child restraints already exist but to the best of our knowledge, there has been no qualitative evidence syntheses on the facilitators and barriers to child restraint usage. This review aims to fill that gap.

\section{Methods}

We searched for qualitative studies, which focussed on perceptions, values and experiences of children, parents/caregivers or any other relevant stakeholders on the use of restraints for children travelling in motor vehicles in PubMed, EMBASE and Global Health and screened reference lists of all included studies. We assessed the quality of included studies with the Critical Appraisal Skills Programme (CASP) checklist and used the PROGRESS Plus lens for an equity focussed analysis.

\section{Results}

We identified a total of 335 records from searching the databases and 5 records from other sources. After screening, we identified 17 studies that met our inclusion criteria. All but one study (which had children as participants) focussed on the perceptions, attitudes, and barriers of parents or caregivers. 
The included studies were from three high-income $(n=14)$ and one upper-middle income $(n=3)$ country. In addition, although many focussed exclusively on participants from culturally and linguistically diverse minorities, the issue of equity was not well addressed. Five major themes emerged from the analysis: (1) Perceived risk for injuries and perceived safety benefits of child restraint usage varies in different settings and between different types of caregivers; (2) Practical issues around the use of child restraints is a major barrier to its uptake as a child safety measure; (3) Restraint use is considered as a mechanism to discipline children rather than as a safety device by parents and as children became older they actively seek opportunities to negotiate the non-usage of restraints; (4) Adoption and enforcement of laws shape perceptions and usage in all settings; and (5) Perceptions and norms of child safety differ among culturally and linguistically diverse groups.

\section{Conclusion}

The results of this systematic review should be considered when designing interventions to promote the uptake of child restraints. However, there is a need to conduct qualitative research around the facilitators and barriers to child restraint usage in low- and middle-income countries. Furthermore, there is a need for more evidence conducted in semi-urban and rural areas and to involve fathers, policy makers, implementers and enforcement agencies in such studies.

\section{Keywords}

Road traffic injury, child restraint systems, seatbelt, child seats, injury prevention

\section{Background}

Death, disability and economic burden due to road traffic injuries are a major public health problem globally and are expected to increase for at least the next two decades ${ }^{1}$. Children contribute a significant proportion of the burden ${ }^{2}$ and so addressing child road traffic injury risk factors is crucial.

Increasing the use of child restraints is one of five key behavioural factors being tracked globally that is aimed at reducing the burden of road injury and is the only one specific to children ${ }^{2}$. In addition, Target 8 of the Global Voluntary performance Targets for Road Safety, which was adopted by United Nations Member States through UN GA resolution 72/271 in April 2018, specifically aims to increase the use of standard child restraint systems to $100 \%$ by $2030^{3}$. As of 2016 only 33 countries, almost all of them highincome countries, had adopted best practice legislation in relation to the use of child restraints 2 .

Ensuring the use of child restraints has been a major challenge despite the legal requirements in these countries ${ }^{4-9}$. While systematic reviews on the effectiveness of various interventions to promote the use of child restraints have already been conducted 10 there has not been a synthesis of evidence to understand the facilitators and barriers to the use of child restraints. The current study, therefore, aims to synthesize qualitative evidence on the barriers to and facilitators of the use of restraints for children travelling in motor vehicles. To the best of our knowledge this is the first qualitative evidence synthesis on the topic and will provide important insights to understand the implementation of best practices and to improve the design of programmes to increase child restraint usage. 


\section{Methods:}

While there are several approaches to qualitative evidence synthesis, the current review broadly follows the principles and guidance laid down in a recent series of papers by the Cochrane Qualitative and Implementation Methods Group ${ }^{11-16}$. The protocol for the study was registered prospectively in PROSPERO (CRD42018102497).

\section{Theoretical Approach}

Our approach in this review is from the "critical realism" standpoint with a pragmatic lens. Critical Realism is a philosophical standpoint which recognises that an observable behaviour - in this instance the use of a child restraint - is influenced by unseen phenomena such as values and expectations. While child restraint use is observable, the use of child restraints is not independent of values, attitudes, prior experiences, capacities and motivations. As such through this philosophical standpoint, and wide qualitative evidence synthesis, we endeavoured to understand the diverse and multi-faceted external reality in all its complexity and depth through perceptions and interpretations reported in individual qualitative studies. A pragmatic lens allows us to include individual qualitative studies irrespective of their ontological stance.

\section{Criteria for considering studies for this review}

\section{Type of studies}

We included primary studies that have used qualitative approaches for both data collection and data analysis. We recognised focus groups, individual in-depth interviews, ethnographic interviews, participant observation, diaries and other narrative methods of data collection as valid tools for qualitative data collection. We also recognised narrative analysis, thematic analysis, grounded theory, phenomenological approaches and discourse analysis as valid methods for qualitative analyses. We excluded studies that used qualitative methods for data collection but did not analyse the data qualitatively. We included qualitative studies irrespective of whether or not they were conducted in conjunction with the implementation of interventions for the use of restraints for children travelling in motor vehicles. We included mixed methods studies only when both the methods and results for the qualitative component were reported separately.

\section{Participants}

We included studies that focussed on the perceptions, values and experiences of children; parents and/ or caregivers; and any other relevant stakeholder involved in formulating policies or programmes or implementing child restraint programmes.

\section{Setting}

We included studies from anywhere in the world and in any setting provided that they met our inclusion criteria.

\section{Types of phenomena of interest}


We included studies where the primary focus were perceptions, attitudes and experiences of parents/ caregivers and children and other stakeholders towards restraints for children travelling in motor vehicles.

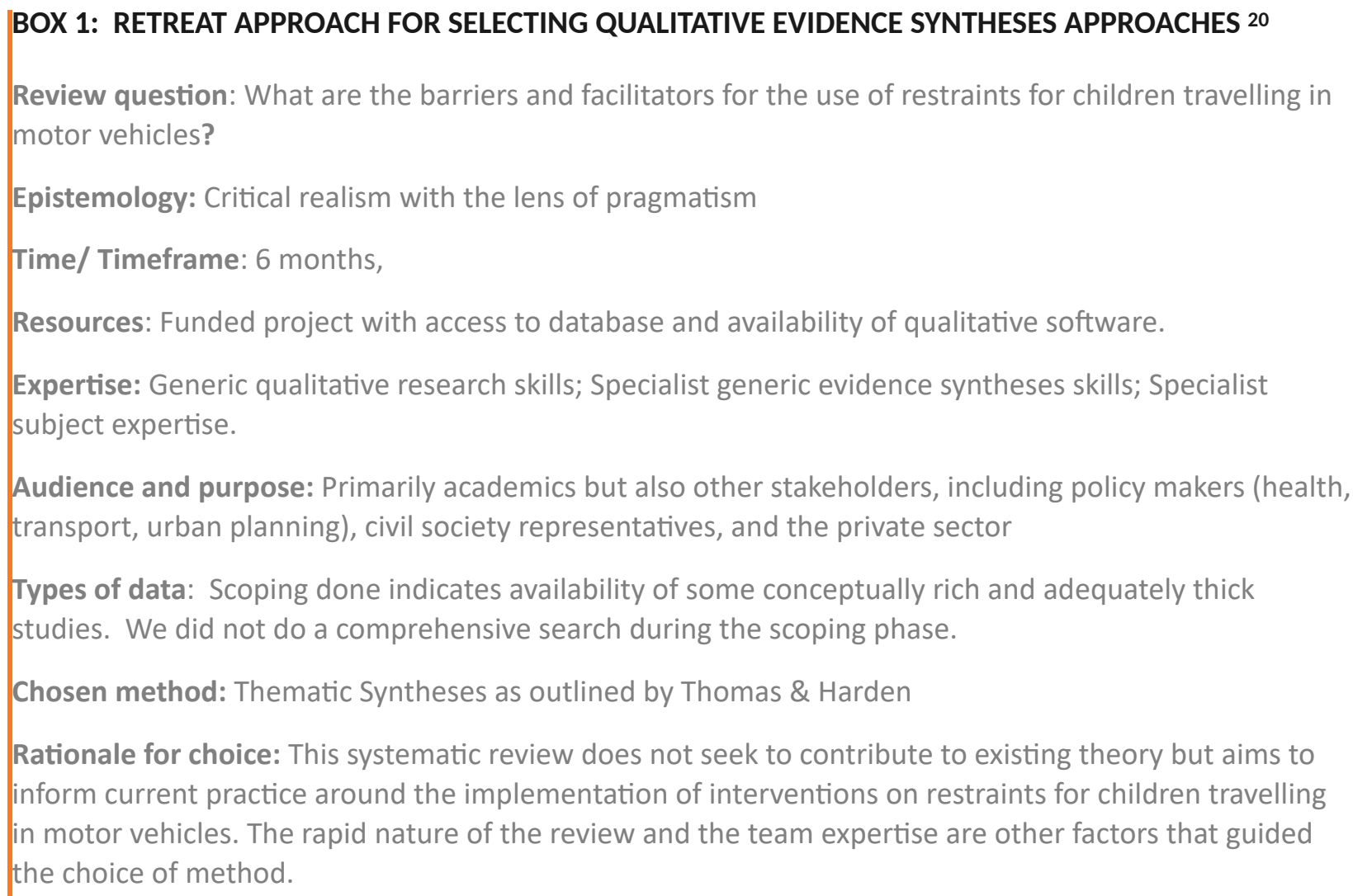

Searching for qualitative evidence

We searched PubMed, EMBASE (EMBASE Classic 1947 to 1973 \& EMBASE 1974 to 2018 July 26) and Global Health for eligible studies using search strategies developed for this purpose (Appendix 1) and updated up to $27^{\text {th }}$ July 2018 . We also searched the reference lists of all included studies found by electronic database search. The search was not restricted by language.

\section{Selection of studies}

In the first phase, one review author (SB) independently assessed eligibility of primary studies based on titles and abstracts. We then acquired the full text of all papers identified as potentially relevant by at least one review author for independent review by two authors (SB, MP). We did not contact study authors for any further information because of pragmatic reasons and timelines.

\section{Data extraction}

We extracted data from studies using an extraction form that was developed iteratively. We extracted data on context, participants, study design and methods in addition to what was required for the quality appraisal of included studies and for thematic analyses. We extracted information on participants using 
the PROGRESS Plus framework. The framework (in the form of an aide-memoire) is used to explicitly understand factors that lead to health inequity. It assesses place of residence, race/ethnicity/culture/ language, occupation, gender/sex, religion, socio-economic status, social capital plus additional factors that may indicate disadvantage 17 .

\section{Assessment of the quality of the included qualitative studies}

We appraised the quality of the included qualitative studies by using the Critical Appraisal Skills Programme (CASP) quality assessment tool for qualitative studies 18 . The tool, which has been recommended for use by the Cochrane Qualitative \& Implementation Methods Group, comprises 10 questions (applied in Table 2) and has been previously used in several other qualitative evidence syntheses studies.

\section{Syntheses Methodology}

We used thematic synthesis as defined by Thomas and Harden 2008 for the purpose of qualitative evidence syntheses ${ }^{19}$. Box 1 presents the detailed rationale for the choice of thematic syntheses as defined in the RETREAT framework 20.

We followed the standard methods outlined by the thematic approach wherein the entire text labelled as 'results' or 'findings' in study reports was used verbatim for further analyses. Broadly, this consisted of the following steps:

- Coding text and developing descriptive themes. The review author conducted line-by-line coding using NVIVO in a set of three articles and developed a hierarchical coding framework and then applied this to newer articles. After every third article, the coding framework was revised iteratively, based on the identification of newer concepts. The final coding framework developed as an output of the iterative process was then applied to all included studies. Repeat checks, constant comparison and discussion between the reviewers was undertaken to ensure consistency. Similarities and differences between codes were constantly compared to develop descriptive themes.

- Development of analytical themes. In the final level of synthesis, we developed higher-level analytical themes by inferring barriers and facilitators for the use of restraints for children travelling in motor vehicles going beyond the interpretations being offered by individual primary studies.

Differences in protocol and the full qualitative evidence synthesis are described in Appendix 2.

\section{Results}

\section{Results of the search}

We identified a total of 335 records from the databases and 5 records from other sources. After removing 90 duplicates, we screened 250 articles based on their titles and abstracts only. We considered 24 full-text papers for inclusion in this synthesis and found 17 studies that met our inclusion criteria (Figure 1). 


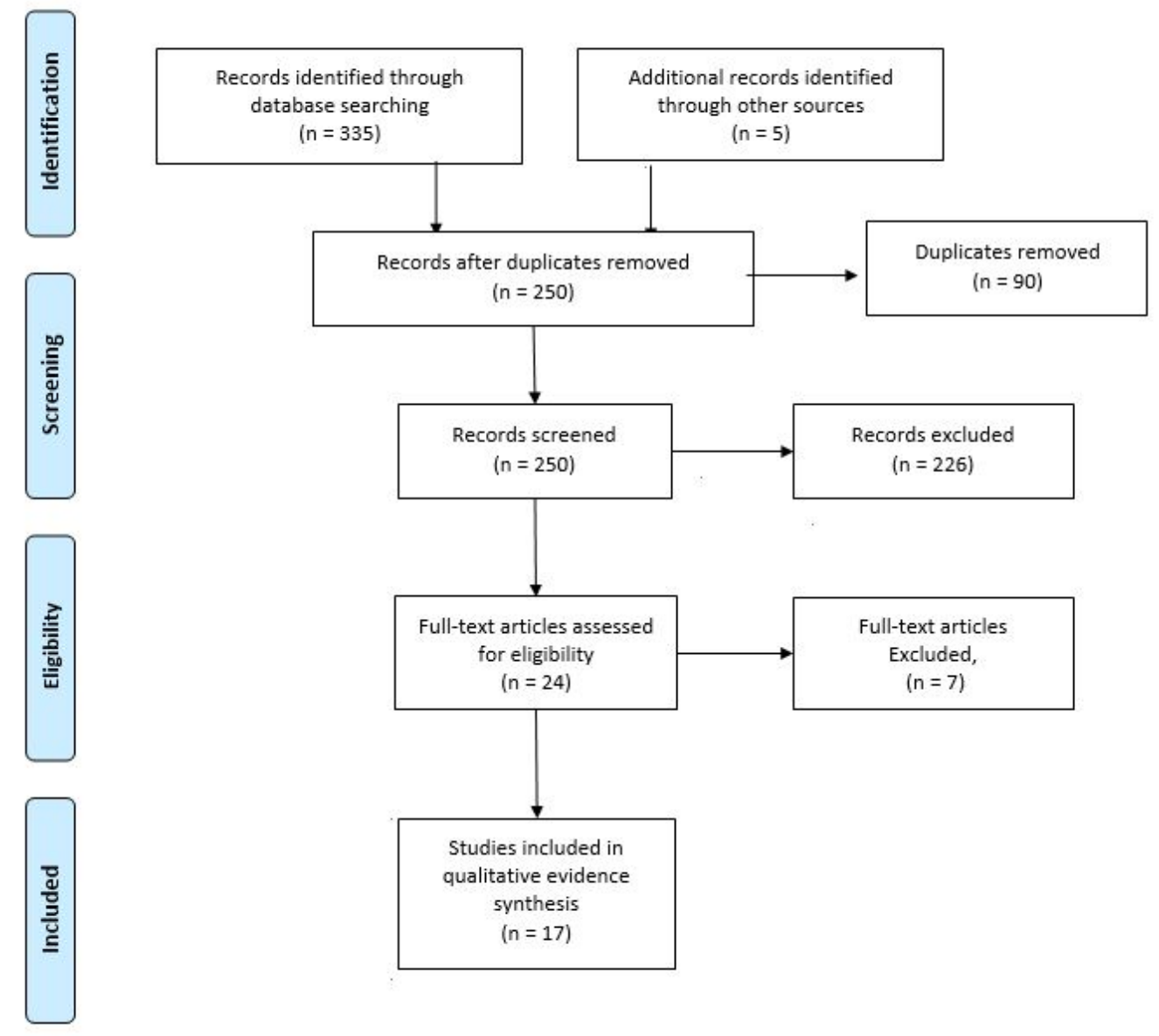

Figure 1 : PRISMA flowchart of the qualitative evidence syntheses

Description of included studies

Characteristics of the included studies is summarised in Table 1 and the details are narratively summarised in subsequent sections. 
Table 1: Characteristics of Included studies 


\begin{tabular}{|c|c|c|c|c|c|c|c|}
\hline $\begin{array}{l}\text { Study } \\
\text { (Country }\end{array}$ & Aim & $\begin{array}{l}\text { Study Design } \\
\text { Summary }\end{array}$ & $\begin{array}{l}\text { Participant } \\
\text { Type }\end{array}$ & $\begin{array}{l}\text { Types of } \\
\text { phenomen } \\
\text { a of } \\
\text { interest }\end{array}$ & $\begin{array}{l}\text { Sampling } \\
\text { frame, \& } \\
\text { recruitment }\end{array}$ & $\begin{array}{l}\text { Method of } \\
\text { data } \\
\text { collection }\end{array}$ & $\begin{array}{l}\text { Method of } \\
\text { data } \\
\text { analyses }\end{array}$ \\
\hline $\begin{array}{l}\text { Rivara } 2001 \\
21 \\
\text { (USA) }\end{array}$ & $\begin{array}{l}\text { To explore } \\
\text { parental } \\
\text { knowledg } \\
\text { e, } \\
\text { attitudes, } \\
\text { beliefs, } \\
\text { and } \\
\text { barriers to } \\
\text { use of } \\
\text { booster } \\
\text { seats in } \\
\text { cars for 4- } \\
8 \text { year old } \\
\text { children. }\end{array}$ & $\begin{array}{l}\text { Focus Group } \\
\text { Discussion } \\
\text { (FGD) } \\
\text { (three). }\end{array}$ & $\begin{array}{l}\text { Parents of } \\
\text { Children } \\
\text { aged } \leq 10 \\
\text { years } \\
(n=30) .\end{array}$ & $\begin{array}{l}\text { Knowledge, } \\
\text { attitudes, } \\
\text { beliefs, and } \\
\text { barriers. }\end{array}$ & $\begin{array}{l}\text { Recruited } \\
\text { through fliers } \\
\text { at local child } \\
\text { and day care } \\
\text { facilities. } \\
\text { Participants } \\
\text { were paid } \\
\$ 30 \text { cash and } \\
\text { given a } \$ 15 \\
\text { gift } \\
\text { certificate. }\end{array}$ & $\begin{array}{l}\text { Not } \\
\text { adequately } \\
\text { reported. } \\
\text { FGDs were 2- } \\
\text { hour } \\
\text { duration. }\end{array}$ & $\begin{array}{l}\text { The results } \\
\text { of the } \\
\text { FGDs were } \\
\text { examined } \\
\text { using the } \\
\text { PRECEDE- } \\
\text { PROCEED } \\
\text { model of } \\
\text { Green and } \\
\text { Kreuter.22 }\end{array}$ \\
\hline
\end{tabular}




\begin{tabular}{|c|c|c|c|c|c|c|c|}
\hline $\begin{array}{l}\text { Simpson } \\
200223 \\
\text { (USA) }\end{array}$ & $\begin{array}{l}\text { To identify } \\
\text { barriers to } \\
\text { booster } \\
\text { seat use } \\
\text { and to } \\
\text { identify } \\
\text { potential } \\
\text { strategies } \\
\text { to } \\
\text { increase } \\
\text { the use of } \\
\text { booster } \\
\text { seats } \\
\text { among } \\
\text { young } \\
\text { preschool } \\
\text { - and } \\
\text { school- } \\
\text { aged } \\
\text { children. }\end{array}$ & $\begin{array}{l}\text { FGD and In- } \\
\text { depth } \\
\text { interviews } \\
\text { (IDI) in two } \\
\text { phases. }\end{array}$ & $\begin{array}{l}\text { Parents and } \\
\text { children } \\
\text { ( } \mathrm{n}=111 \text { ) } \\
\text { Participant } \\
\text { groupings } \\
\text { were } \\
\text { determined } \\
\text { according } \\
\text { to the age } \\
\text { of the child, } \\
\text { method of } \\
\text { restraint. } \\
\text { Those who } \\
\text { had } \\
\text { participate } \\
\text { d in any } \\
\text { FGD in the } \\
\text { last } 3 \\
\text { months or } \\
\text { were } \\
\text { employees } \\
\text { of the firm } \\
\text { which } \\
\text { conducted } \\
\text { this were } \\
\text { excluded. }\end{array}$ & $\begin{array}{l}\text { Perceptions } \\
\text { and } \\
\text { experiences } \\
\text {. }\end{array}$ & $\begin{array}{l}\text { Phase I } \\
\text { ( } n=54): 31 \\
\text { parents in } 3 \\
\text { FGD }+8 \\
\text { children in } 2 \\
\text { FGD + } 15 \\
\text { parents in } \\
\text { IDIs. Phase II } \\
\text { ( } n=57): 36 \\
\text { parents in } 4 \\
\text { FGD + } 21 \\
\text { parents in } \\
\text { telephone } \\
\text { discussions. } \\
\text { Recruited by } \\
\text { local market } \\
\text { research } \\
\text { firms } \\
\text { randomly } \\
\text { chosen from } \\
\text { databases. } \\
\text { Eligible } \\
\text { candidates } \\
\text { received an } \\
\text { honorarium } \\
\text { for } \\
\text { participation. }\end{array}$ & $\begin{array}{l}\text { Moderator } \\
\text { introduced } \\
\text { the FGD } \\
\text { process and } \\
\text { the specific } \\
\text { topic for the } \\
\text { group. } \\
\text { Sessions } \\
\text { were } \\
\text { audiotaped } \\
\text { and } \\
\text { videotaped } \\
\text { and } \\
\text { observed } \\
\text { through a 1- } \\
\text { way mirror } \\
\text { by } 2 \\
\text { physicians, a } \\
\text { psychologist } \\
\text { and a } \\
\text { qualitative } \\
\text { research } \\
\text { specialist. }\end{array}$ & $\begin{array}{l}\text { Experts, } \\
\text { the } \\
\text { moderator, } \\
\text { and a } \\
\text { research } \\
\text { assistant } \\
\text { conducted } \\
\text { data } \\
\text { analysis. } \\
\text { Transcripts } \\
\text { were } \\
\text { reviewed } \\
\text { for } \\
\text { common } \\
\text { themes } \\
\text { and new } \\
\text { ideas. } \\
\text { After each } \\
\text { phase of } \\
\text { FGDs and } \\
\text { IDIs, the } \\
\text { moderator } \\
\text { drafted an } \\
\text { initial } \\
\text { summary } \\
\text { for review. } \\
\text { All } \\
\text { researcher } \\
\text { s who } \\
\text { observed } \\
\text { the FGDs } \\
\text { reviewed } \\
\text { the } \\
\text { summary } \\
\text { and made } \\
\text { comments } \\
\text { to reach } \\
\text { consensus. } \\
\text { All possible } \\
\text { interpreta } \\
\text { tions were } \\
\text { recorded } \\
\text { when no } \\
\text { consensus } \\
\text { was } \\
\text { reached. }\end{array}$ \\
\hline
\end{tabular}




\begin{tabular}{|c|c|c|c|c|c|c|c|}
\hline $\begin{array}{l}\text { Lee } 200324 \\
\text { (USA) }\end{array}$ & $\begin{array}{l}\text { To } \\
\text { investigat } \\
\text { e Latino } \\
\text { parents' } \\
\text { knowledg } \\
\text { e, } \\
\text { attitudes } \\
\text { and } \\
\text { beliefs } \\
\text { about } \\
\text { booster } \\
\text { seats, } \\
\text { barriers to } \\
\text { booster } \\
\text { seat use, } \\
\text { and } \\
\text { effective } \\
\text { strategies } \\
\text { for } \\
\text { message } \\
\text { delivery in } \\
\text { the Latino } \\
\text { communit } \\
\text { y. }\end{array}$ & FGDs (two). & $\begin{array}{l}\text { Parents } \\
\text { were } \\
\text { eligible if } \\
\text { they were } \\
\text { Spanish- } \\
\text { speaking, } \\
\text { had } \\
\text { children } \\
\text { under the } \\
\text { age of } 10 \\
\text { years, and } \\
\text { owned or } \\
\text { drove a car. }\end{array}$ & $\begin{array}{l}\text { Knowledge } \\
\text { attitudes } \\
\text { and beliefs. }\end{array}$ & $\begin{array}{l}\text { Recruited by } \\
\text { a member of } \\
\text { the research } \\
\text { team, who } \\
\text { went to two } \\
\text { community } \\
\text { centres in } \\
\text { areas with } \\
\text { high Latino } \\
\text { population in } \\
\text { Seattle. } \\
\text { There were } \\
23 \\
\text { participants } \\
\text { (12 in the } \\
\text { first group } \\
\text { and } 11 \text { in the } \\
\text { second). }\end{array}$ & $\begin{array}{l}\text { Not much is } \\
\text { reported - } \\
\text { article only } \\
\text { mentions } \\
\text { what topics } \\
\text { were } \\
\text { discussed. }\end{array}$ & $\begin{array}{l}\text { Only } \\
\text { mentions } \\
\text { that the } \\
\text { results of } \\
\text { the FGDs } \\
\text { were } \\
\text { audiotape } \\
\text { d and } \\
\text { reviewed } \\
\text { by the } \\
\text { authors to } \\
\text { identify } \\
\text { important } \\
\text { themes. }\end{array}$ \\
\hline
\end{tabular}




\begin{tabular}{|c|c|c|c|c|c|c|c|}
\hline $\begin{array}{l}\text { Agran } 2004 \\
25 \\
\text { (USA) }\end{array}$ & $\begin{array}{l}\text { To } \\
\text { examine } \\
\text { the } \\
\text { factors } \\
\text { contributi } \\
\text { ng to non- } \\
\text { use or } \\
\text { part-time } \\
\text { use of a } \\
\text { child } \\
\text { restraints } \\
\text { and the } \\
\text { effects of } \\
\text { exposure } \\
\text { to a } \\
\text { violator } \\
\text { class for } \\
\text { those } \\
\text { cited for } \\
\text { violation } \\
\text { of the } \\
\text { California } \\
\text { Child } \\
\text { Passenger } \\
\text { Safety } \\
\text { (CPS) law. }\end{array}$ & $\begin{array}{l}\text { FGDs and } \\
\text { results were } \\
\text { used to } \\
\text { guide the } \\
\text { development } \\
\text { of a study of } \\
\text { an existing } \\
\text { violator class } \\
\text { for CPS law. }\end{array}$ & $\begin{array}{l}3 \text { FGDs } \\
\text { included } 24 \\
\text { individuals } \\
\text { who had } \\
\text { been cited } \\
\text { for } \\
\text { violation of } \\
\text { the } \\
\text { California } \\
\text { CPS law }\end{array}$ & $\begin{array}{l}\text { Not } \\
\text { specified. }\end{array}$ & $\begin{array}{l}\text { Recruited } \\
\text { from } \\
\text { violators } \\
\text { registering } \\
\text { for a class } \\
\text { required by } \\
\text { several } \\
\text { courts in Los } \\
\text { Angeles } \\
\text { County. }\end{array}$ & $\begin{array}{l}2 \text { FGDs in } \\
\text { English and } 1 \\
\text { in Spanish. } \\
\text { Each of } 90 \\
\text { minutes } \\
\text { duration just } \\
\text { before the } \\
\text { violator } \\
\text { class. } \\
\text { Facilitators } \\
\text { familiar with } \\
\text { child } \\
\text { occupant } \\
\text { protection } \\
\text { and child } \\
\text { development } \\
\text { led the } \\
\text { groups. }\end{array}$ & $\begin{array}{l}\text { An } \\
\text { observer, } \\
\text { supplemen } \\
\text { ted by } \\
\text { audiotapes } \\
\text { of the } \\
\text { sessions, } \\
\text { conducted } \\
\text { a thematic } \\
\text { analysis of } \\
\text { notes. The } \\
\text { University } \\
\text { of } \\
\text { California } \\
\text { institution } \\
\text { al review } \\
\text { board } \\
\text { approved } \\
\text { the } \\
\text { protocol. }\end{array}$ \\
\hline $\begin{array}{l}\text { Lennon } \\
200726 \\
\text { (Australia) }\end{array}$ & $\begin{array}{l}\text { To explore } \\
\text { parental } \\
\text { perceptio } \\
\text { ns of } \\
\text { barriers to } \\
\text { placing } \\
\text { their } \\
\text { children } \\
\text { in the rear } \\
\text { seat of } \\
\text { passenger } \\
\text { vehicles } \\
\text { whenever } \\
\text { possible. }\end{array}$ & $\begin{array}{l}\text { Interpretive } \\
\text { phenomenol } \\
\text { ogical } \\
\text { qualitative } \\
\text { approach } \\
\text { with FGDs } \\
\text { being held } \\
\text { with urban } \\
\text { parent- } \\
\text { driver. }\end{array}$ & $\begin{array}{l}\text { Parents of } \\
\text { children } \\
\text { aged }>12 \\
\text { years who } \\
\text { regularly } \\
\text { drive their } \\
\text { children in } \\
\text { passenger } \\
\text { vehicles } \\
\text { (with a rear } \\
\text { seat). } \\
24 \text { parents } \\
\text { participate } \\
\text { d in } 5 \\
\text { separate } \\
\text { groups. }\end{array}$ & Perception. & $\begin{array}{l}\text { Parents of } \\
\text { children aged } \\
>12 \text { years } \\
\text { who } \\
\text { regularly } \\
\text { drive their } \\
\text { children in } \\
\text { passenger } \\
\text { vehicles } \\
\text { (with a rear } \\
\text { seat) were } \\
\text { recruited by } \\
\text { personal } \\
\text { approach in } \\
\text { the open-air } \\
\text { carparks of } \\
\text { two urban } \\
\text { shopping } \\
\text { centres in } \\
\text { Brisbane, } \\
\text { Australia. }\end{array}$ & $\begin{array}{l}\text { A semi- } \\
\text { structured } \\
\text { discussion } \\
\text { schedule was } \\
\text { used. Except } \\
\text { for one } \\
\text { group where } \\
\text { notes were } \\
\text { taken, } \\
\text { discussions } \\
\text { were } \\
\text { transcribed } \\
\text { by a } \\
\text { professional } \\
\text { stenographer } \\
\text { to allow the } \\
\text { accurate } \\
\text { recall of } \\
\text { exact terms } \\
\text { used by } \\
\text { parents. }\end{array}$ & $\begin{array}{l}\text { Transcripts } \\
\text { and } \\
\text { discussion } \\
\text { notes were } \\
\text { analysed } \\
\text { using QSR } \\
\text { NVivo 2.0. } \\
\text { Thematic } \\
\text { analysis, } \\
\text { following } \\
\text { the } \\
\text { process } \\
\text { described } \\
\text { by van } \\
\text { Manen } 27 \\
\text { until data } \\
\text { saturation } \\
\text { was } \\
\text { achieved. }\end{array}$ \\
\hline
\end{tabular}




\begin{tabular}{|c|c|c|c|c|c|c|c|}
\hline $\begin{array}{l}\text { Medoff- } \\
\text { Cooper } \\
200728 \\
\text { (USA) }\end{array}$ & $\begin{array}{l}\text { To } \\
\text { examine } \\
\text { the } \\
\text { beliefs, } \\
\text { behaviour } \\
\text { s, barriers } \\
\text { and } \\
\text { motivatin } \\
\text { g factors } \\
\text { for use of } \\
\text { child car } \\
\text { seats } \\
\text { among } \\
\text { children } \\
\text { aged } 3 \text { to } \\
7 \text { years. }\end{array}$ & $\begin{array}{l}\text { FGDs and } \\
\text { IDIs. }\end{array}$ & $\begin{array}{l}\text { Mothers } \\
\text { (16 in } 2 \\
\text { FGDs) } \\
\text { owning a } \\
\text { car and } \\
\text { having a } \\
\text { child } \\
\text { between } \\
\text { the ages of } \\
3 \text { and } 7 \\
\text { who rode } \\
\text { in the car at } \\
\text { least once a } \\
\text { week. }\end{array}$ & $\begin{array}{l}\text { Beliefs, } \\
\text { behaviours, } \\
\text { perceived } \\
\text { barriers, } \\
\text { and } \\
\text { motivations } \\
\text {. }\end{array}$ & $\begin{array}{l}\text { Participants } \\
\text { were } \\
\text { recruited } \\
\text { from a } \\
\text { marketing } \\
\text { data base of } \\
\text { individuals } \\
\text { who met the } \\
\text { sampling } \\
\text { criteria of } \\
\text { owning a car } \\
\text { and having a } \\
\text { child } \\
\text { between the } \\
\text { ages of } 3 \text { and } \\
7 \text { who rode } \\
\text { in the car at } \\
\text { least once a } \\
\text { week. } \\
\text { Institutional } \\
\text { Review } \\
\text { Board } \\
\text { approval was } \\
\text { obtained } \\
\text { before the } \\
\text { study. } \\
\text { Written } \\
\text { consent was } \\
\text { obtained } \\
\text { from all } \\
\text { participants. }\end{array}$ & $\begin{array}{l}\text { FGDs had a } \\
\text { professional } \\
\text { group leader } \\
\text { with a } \\
\text { graduate } \\
\text { degree in } \\
\text { clinical } \\
\text { psychology. } \\
\text { The in-depth } \\
\text { interviewing } \\
\text { of } \\
\text { respondents } \\
\text { was done } \\
\text { with skill in a } \\
\text { dynamic } \\
\text { atmosphere } \\
\text { of rapport, } \\
\text { sensitivity, } \\
\text { and } \\
\text { openness to } \\
\text { sharing } \\
\text { thoughts and } \\
\text { feelings. The } \\
\text { interview } \\
\text { guide } \\
\text { contained } \\
\text { open-ended } \\
\text { questions. }\end{array}$ & $\begin{array}{l}\text { The } \\
\text { analysis } \\
\text { came from } \\
\text { understan } \\
\text { ding and } \\
\text { interpretin } \\
\text { g the } \\
\text { verbal, } \\
\text { visual, and } \\
\text { other } \\
\text { inputs } \\
\text { from } \\
\text { responden } \\
\text { ts and } \\
\text { client- } \\
\text { relevant } \\
\text { themes. } \\
\text { The } \\
\text { moderator } \\
\text { using the } \\
\text { topics } \\
\text { identified } \\
\text { in advance } \\
\text { as the } \\
\text { areas of } \\
\text { interest } \\
\text { completed } \\
\text { an in- } \\
\text { depth } \\
\text { report. }\end{array}$ \\
\hline
\end{tabular}




\begin{tabular}{|c|c|c|c|c|c|c|c|}
\hline $\begin{array}{l}\text { Winston } \\
200729 \\
\text { (USA) }\end{array}$ & $\begin{array}{l}\text { 1. To } \\
\text { identify } \\
\text { factors } \\
\text { that } \\
\text { influence } \\
\text { parents' } \\
\text { current } \\
\text { child } \\
\text { restraint } \\
\text { use } \\
\text { behaviour } \\
\text { s and } \\
\text { intentions } \\
\text { for future } \\
\text { use, and } \\
2 \text {. To test } \\
\text { interventi } \\
\text { ons that } \\
\text { address } \\
\text { these } \\
\text { factors as } \\
\text { a means } \\
\text { to } \\
\text { promote } \\
\text { appropria } \\
\text { te } \\
\text { restraint } \\
\text { use } \\
\text { behaviour } \\
\text { s, } \\
\text { particularl } \\
\text { y the use } \\
\text { of belt- } \\
\text { positionin } \\
\text { g booster } \\
\text { seats. }\end{array}$ & $\begin{array}{l}3 \text { phases: } \\
\text { Formative } \\
\text { Phase - FGDs } \\
\text { defined } \\
\text { barriers; } \\
\text { Developmen } \\
\text { t Phase - } \\
\text { literature } \\
\text { review to } \\
\text { identify } \\
\text { interventions } \\
\text { and in the } \\
\text { Evaluative } \\
\text { Phase - } 4 \\
\text { existing and } \\
3 \text { new } \\
\text { interventions } \\
\text { were } \\
\text { evaluated. } \\
\text { There was } \\
\text { also a follow- } \\
\text { up } \\
\text { telephonic } \\
\text { survey. }\end{array}$ & $\begin{array}{l}\text { Parents of } \\
\text { children } \\
\text { between } \\
\text { the ages of } \\
3 \text { and } 8 \\
\text { years who } \\
\text { never or } \\
\text { rarely used } \\
\text { appropriate } \\
\text { restraints. } \\
\text { Additional } \\
\text { inclusion } \\
\text { criteria } \\
\text { included: } \\
\text { educational } \\
\text { attainment } \\
\text { of, at most, } \\
\text { a high } \\
\text { school } \\
\text { diploma, } \\
\text { and travel } \\
\text { with } \\
\text { children in } \\
\text { motor } \\
\text { vehicles at } \\
\text { least once a } \\
\text { week } \\
\text { Formative } \\
\text { phase: } 10 \\
\text { FGDs, } n= \\
117 \\
\text { Evaluative } \\
\text { phase: } 20 \\
\text { FGDs, } \\
\mathrm{n}=171\end{array}$ & $\begin{array}{l}\text { Beliefs and } \\
\text { behaviours. }\end{array}$ & $\begin{array}{l}\text { Study } \\
\text { participants } \\
\text { were } \\
\text { recruited in } \\
\text { both phases } \\
\text { using } \\
\text { telephone } \\
\text { calls and } \\
\text { printed } \\
\text { flyers, } \\
\text { through an } \\
\text { existing } \\
\text { injury free } \\
\text { children } \\
\text { network. All } \\
\text { participants } \\
\text { were offered } \\
\text { reading } \\
\text { assistance } \\
\text { and/or } \\
\text { Spanish } \\
\text { language } \\
\text { translation, } \\
\text { according to } \\
\text { the } \\
\text { participant's } \\
\text { preference. } \\
\text { The } \\
\text { Institutional } \\
\text { Review } \\
\text { Board at The } \\
\text { Children's } \\
\text { Hospital of } \\
\text { Philadelphia } \\
\text { approved all } \\
\text { recruitment } \\
\text { and consent } \\
\text { procedures, } \\
\text { as well as all } \\
\text { study } \\
\text { protocols } \\
\text { and surveys. }\end{array}$ & $\begin{array}{l}\text { Discussion } \\
\text { guides } \\
\text { prepared for } \\
\text { both } \\
\text { Formative } \\
\text { and } \\
\text { Evaluative } \\
\text { phases. In } \\
\text { Evaluative } \\
\text { phase the } \\
\text { parent's } \\
\text { reactions to } \\
\text { interventions } \\
\text { were } \\
\text { assessed } \\
\text { after they } \\
\text { were } \\
\text { provided } \\
\text { with free } \\
\text { belt- } \\
\text { positioning } \\
\text { booster seats } \\
\text { as well as } \\
\text { education on } \\
\text { their use. } \\
\text { Follow-up } \\
\text { interviews } \\
\text { were } \\
\text { conducted } 6 \\
\text { weeks later. }\end{array}$ & $\begin{array}{l}\text { FGDs were } \\
\text { taped and } \\
\text { transcribed } \\
\text { Transcripts } \\
\text { were } \\
\text { coded, and } \\
\text { thematic } \\
\text { analyses } \\
\text { done } 2 \text { by } \\
\text { research } \\
\text { assistants } \\
\text { under the } \\
\text { supervisio } \\
\text { n of the } \\
\text { study } \\
\text { coordinato } \\
\text { r. } \\
\text { When } \\
\text { consensus } \\
\text { on } \\
\text { interpreta } \\
\text { tions was } \\
\text { not } \\
\text { reached all } \\
\text { interpreta } \\
\text { tions were } \\
\text { included in } \\
\text { the } \\
\text { summary } \\
\text { document. }\end{array}$ \\
\hline
\end{tabular}




\begin{tabular}{|c|c|c|c|c|c|c|c|}
\hline $\begin{array}{l}\text { Johnston } \\
200930 \\
\text { (USA) }\end{array}$ & $\begin{array}{l}\text { To expand } \\
\text { our } \\
\text { understan } \\
\text { ding of } \\
\text { the } \\
\text { determina } \\
\text { nts of } \\
\text { booster } \\
\text { seat use } \\
\text { with a } \\
\text { broader } \\
\text { range of } \\
\text { families } \\
\text { from our } \\
\text { priority } \\
\text { populatio } \\
\mathrm{n} \text { (low- } \\
\text { income } \\
\text { African } \\
\text { American, } \\
\text { Somali, } \\
\text { and } \\
\text { Vietname } \\
\text { se } \\
\text { parents). }\end{array}$ & FGDs & $\begin{array}{l}\text { African } \\
\text { American, } \\
\text { Somali, and } \\
\text { Vietnamese } \\
\text { parents or } \\
\text { custodial } \\
\text { grandparen } \\
\text { ts of } \\
\text { children } \\
\text { between } \\
\text { birth and } 9 \\
\text { years of } \\
\text { age and } \\
\text { reported } \\
\text { that they } \\
\text { "did not } \\
\text { always" use } \\
\text { a booster } \\
\text { seat when } \\
\text { their } \\
\text { booster- } \\
\text { eligible } \\
\text { child was } \\
\text { riding in a } \\
\text { car. There } \\
\text { were } 26 \\
\text { participants } \\
\text { across } 3 \\
\text { FGDs. }\end{array}$ & $\begin{array}{l}\text { Not } \\
\text { specified. }\end{array}$ & $\begin{array}{l}\text { Participants } \\
\text { were } \\
\text { recruited } \\
\text { through } \\
\text { posters, } \\
\text { flyers, and } \\
\text { information } \\
\text { booths at } \\
\text { community } \\
\text { clinics, } \\
\text { community } \\
\text { centres, and } \\
\text { other social } \\
\text { service } \\
\text { providers. } \\
\text { Interested } \\
\text { individuals } \\
\text { were pre- } \\
\text { screened to } \\
\text { ensure } \\
\text { eligibility } \\
\text { criteria was } \\
\text { met. All } \\
\text { participants } \\
\text { received } \\
\text { childcare, a } \\
\text { meal, a smal } \\
\text { stipend } \\
\text { (\$25), and } \\
\text { information } \\
\text { on child } \\
\text { passenger } \\
\text { safety at the } \\
\text { conclusion o } \\
\text { FGDs. }\end{array}$ & $\begin{array}{l}\text { FGDs were } \\
\text { conducted } \\
\text { by an } \\
\text { experienced } \\
\text { African } \\
\text { American, } \\
\text { male } \\
\text { facilitator. } \\
\text { Somali and } \\
\text { Vietnamese } \\
\text { sessions } \\
\text { included an } \\
\text { interpreter } \\
\text { who also co- } \\
\text { facilitated } \\
\text { the group. A } \\
\text { semi- } \\
\text { structured } \\
\text { guide was } \\
\text { used. FGDs } \\
\text { were } \\
\text { conducted at } \\
\text { settings } \\
\text { familiar and } \\
\text { comfortable } \\
\text { to the } \\
\text { attendees. }\end{array}$ & $\begin{array}{l}\text { FGDs were } \\
\text { audiotape } \\
d \text {, } \\
\text { transcribed } \\
\text { and } \\
\text { translated. } \\
\text { Three } \\
\text { researcher } \\
\text { s } \\
\text { independe } \\
\text { ntly } \\
\text { reviewed } \\
\text { transcripts } \\
\text { and field } \\
\text { notes } \\
\text { completed } \\
\text { by study } \\
\text { staff. } \\
\text { Major } \\
\text { themes in } \\
\text { each } \\
\text { language } \\
\text { or cultural } \\
\text { group } \\
\text { were } \\
\text { identified } \\
\text { and } \\
\text { categorize } \\
d \\
\text { according } \\
\text { to the } \\
\text { major } \\
\text { domains of } \\
\text { the } \\
\text { theoretical } \\
\text { models. }\end{array}$ \\
\hline
\end{tabular}




\begin{tabular}{|c|c|c|c|c|c|c|c|}
\hline $\begin{array}{l}\text { Erkoboni } \\
2010^{31} \\
\text { (China) }\end{array}$ & $\begin{array}{l}\text { To explore } \\
\text { the } \\
\text { efficacy } \\
\text { and } \\
\text { acceptabil } \\
\text { ity of } \\
\text { using a } \\
\text { US- } \\
\text { developed } \\
\text { belt- } \\
\text { positionin } \\
\text { g booster } \\
\text { seat use } \\
\text { promotio } \\
\mathrm{n} \\
\text { interventi } \\
\text { on in } \\
\text { Beijing. }\end{array}$ & $\begin{array}{l}\text { FGDs were } \\
\text { conducted in } \\
\text { relation to an } \\
\text { intervention } \\
\text { (a Chinese- } \\
\text { produced } \\
\text { instructional } \\
\text { video and an } \\
\text { English- } \\
\text { language } \\
\text { video } \\
\text { dubbed into } \\
\text { Mandarin) } \\
\text { with } \\
\text { theoretically } \\
\text { driven } \\
\text { messages } \\
\text { through a } \\
\text { personal } \\
\text { story- } \\
\text { knowledge } \\
\text { only video } \\
\text { versus } \\
\text { knowledge + } \\
\text { motivation } \\
\text { video. }\end{array}$ & $\begin{array}{l}\text { Eligible } \\
\text { parents } \\
\text { were those } \\
\text { who owned } \\
\text { a car and } \\
\text { had } \\
\text { children } \\
\text { between } \\
\text { the ages of } \\
3 \text { and } 8 \\
\text { years } \\
\text { enrolled in } \\
\text { a Beijing } \\
\text { kindergarte } \\
n \text { or } \\
\text { elementary } \\
\text { school. }\end{array}$ & $\begin{array}{l}\text { Parents' } \\
\text { perceived } \\
\text { benefits, } \\
\text { disadvantag } \\
\text { es, } \\
\text { facilitators, } \\
\text { and } \\
\text { barriers. }\end{array}$ & $\begin{array}{l}\text { Parents were } \\
\text { recruited for } \\
\text { the study } \\
\text { through } \\
\text { kindergarten } \\
\text { s and } \\
\text { elementary } \\
\text { schools. } \\
\text { Parents were } \\
\text { informed } \\
\text { about the } \\
\text { study in } \\
\text { school or } \\
\text { parent- } \\
\text { teacher } \\
\text { meetings and } \\
\text { those willing } \\
\text { participated } \\
\text { (five groups, } \\
71 \\
\text { participants). }\end{array}$ & $\begin{array}{l}\text { Collection of } \\
\text { qualitative } \\
\text { data on } \\
\text { target } \\
\text { constructs - } \\
\text { initial FGD. } \\
\text { Collection of } \\
\text { qualitative } \\
\text { data on } \\
\text { reactions to } \\
\text { videos. } \\
\text { Parents were } \\
\text { shown the } \\
\text { two videos } \\
\text { and then } \\
\text { FGD was } \\
\text { conducted } \\
\text { after it. }\end{array}$ & $\begin{array}{l}\text { FGDs were } \\
\text { taped, } \\
\text { transcribed } \\
\text {, and } \\
\text { translated } \\
\text { into } \\
\text { English. } \\
\text { Transcripts } \\
\text { were } \\
\text { coded by } \\
\text { research } \\
\text { staff on } \\
\text { the basis } \\
\text { of the } \\
\text { themes. In } \\
\text { addition, } \\
\text { parent } \\
\text { responses } \\
\text { to the } \\
\text { video } \\
\text { interventio } \\
\text { ns were } \\
\text { coded to } \\
\text { qualitativel } \\
\text { y assess } \\
\text { participant } \\
\text { 's response } \\
\text { to the } \\
\text { programm } \\
\text { es. To } \\
\text { supplemen } \\
\text { t these } \\
\text { transcripts, } \\
\text { field notes } \\
\text { were taken } \\
\text { by } \\
\text { researcher } \\
\text { s present } \\
\text { during the } \\
\text { discussion. } \\
\text { A } \\
\text { translator } \\
\text { was } \\
\text { present } \\
\text { during the } \\
\text { FGD for } \\
\text { each } \\
\text { English- } \\
\text { speaking } \\
\text { researrher }\end{array}$ \\
\hline
\end{tabular}




\begin{tabular}{|c|c|c|c|c|c|c|c|}
\hline $\begin{array}{l}\text { Brown } \\
201332 \\
\text { (Australia) }\end{array}$ & $\begin{array}{l}\text { To } \\
\text { qualitativ } \\
\text { ely } \\
\text { explore } \\
\text { barriers to } \\
\text { optimal } \\
\text { child } \\
\text { restraint } \\
\text { use using } \\
\text { the } \\
\text { integrativ } \\
\text { e } \\
\text { behaviour } \\
\text { change } \\
\text { model in } \\
\text { culturally } \\
\text { and } \\
\text { linguistical } \\
\text { ly diverse } \\
\text { communi } \\
\text { ties in } \\
\text { New } \\
\text { South } \\
\text { Wales, } \\
\text { Australia }\end{array}$ & $\begin{array}{l}11 \text { language } \\
\text { specific FGDs } \\
\text { involving } 71 \\
\text { parents or } \\
\text { grandparents } \\
\text { of children } \\
\text { aged 3-8 } \\
\text { years. }\end{array}$ & $\begin{array}{l}\text { Participants } \\
\text { had to: (1) } \\
\text { speak a } \\
\text { language } \\
\text { other than } \\
\text { English at } \\
\text { home; (2) } \\
\text { be aged } \\
\text { over } 18 \\
\text { years; and } \\
\text { (3) have } \\
\text { travelled in } \\
\text { a car with } \\
\text { at least one } \\
\text { child } \\
\text { between } 3 \\
\text { and } 8 \text { years } \\
\text { old in the } \\
\text { last } 6 \\
\text { months. }\end{array}$ & $\begin{array}{l}\text { Existing } \\
\text { knowledge } \\
\text { and } \\
\text { experience } \\
\text { as well as } \\
\text { underlying } \\
\text { beliefs and } \\
\text { motivations } \\
\text {. }\end{array}$ & $\begin{array}{l}71 \\
\text { participants } \\
\text { for } 11 \text { FGDs. } \\
\text { Recruitment } \\
\text { was done } \\
\text { using } \\
\text { advertiseme } \\
\text { nts in target } \\
\text { languages } \\
\text { through } \\
\text { existing } \\
\text { community } \\
\text { groups or by } \\
\text { direct } \\
\text { invitation } \\
\text { from } \\
\text { community } \\
\text { leaders to } \\
\text { increase } \\
\text { homogeneity } \\
\text { within } \\
\text { groups, and } \\
\text { ensure } \\
\text { participants } \\
\text { were in } \\
\text { comfortable } \\
\text { and familiar } \\
\text { settings to } \\
\text { encourage } \\
\text { free } \\
\text { discussion. }\end{array}$ & $\begin{array}{l}\text { FGDs were } \\
\text { held over a } \\
\text { 2-hr period } \\
\text { and aided by } \\
\text { a semi- } \\
\text { structure } \\
\text { topic guide } \\
\text { which was } \\
\text { not changed } \\
\text { during the } \\
\text { entire study. } \\
\text { A } \\
\text { standardised } \\
\text { questionnair } \\
\text { e was used } \\
\text { to collect } \\
\text { demographic } \\
\text { information. }\end{array}$ & $\begin{array}{l}\text { Content } \\
\text { analysis } \\
\text { using } \\
\text { major } \\
\text { concepts } \\
\text { of } \\
\text { Fishbein's } \\
\text { integrative } \\
\text { behaviour } \\
\text { change } \\
\text { model } 33 \text {. } \\
\text { Initially a } \\
\text { single } \\
\text { researcher } \\
\text { went } \\
\text { through } \\
\text { the } \\
\text { transcripts } \\
\text { to do } \\
\text { content } \\
\text { analysis. } \\
\text { This was } \\
\text { repeated } \\
\text { by a } \\
\text { second } \\
\text { author and } \\
\text { finally two } \\
\text { other } \\
\text { researcher } \\
\text { s reviewed } \\
\text { the final } \\
\text { assignmen } \\
\text { tof } \\
\text { discussion } \\
\text { elements } \\
\text { to the } \\
\text { model } \\
\text { constructs. } \\
\text { Non- } \\
\text { spoken } \\
\text { communic } \\
\text { ation was } \\
\text { not } \\
\text { communic } \\
\text { ated in the } \\
\text { study. }\end{array}$ \\
\hline
\end{tabular}




\begin{tabular}{|c|c|c|c|c|c|c|c|}
\hline $\begin{array}{l}\text { Chen } 2014 \\
34 \\
\text { (China) }\end{array}$ & $\begin{array}{l}\text { To explore } \\
\text { parent } \\
\text { drivers' } \\
\text { perceptio } \\
\text { ns and } \\
\text { experienc } \\
\text { es } \\
\text { regarding } \\
\text { use of } \\
\text { child } \\
\text { safety } \\
\text { restraint, } \\
\text { and to } \\
\text { identify } \\
\text { the } \\
\text { difference } \\
\text { s in the } \\
\text { perceptio } \\
\text { ns and } \\
\text { experienc } \\
\text { es } \\
\text { between } \\
\text { child } \\
\text { safety } \\
\text { restraint } \\
\text { users and } \\
\text { non- } \\
\text { users. }\end{array}$ & $\begin{array}{l}14 \text { IDIs } \\
\text { among } \\
\text { parents with } \\
\text { a child under } \\
\text { the age of } 6 \\
\text { years. Of } 14 \\
\text { parents } \\
\text { interviewed, } \\
7 \text { parents } \\
\text { were child } \\
\text { safety seat } \\
\text { users while } 7 \\
\text { were non- } \\
\text { users. Ages } \\
\text { of parents } \\
\text { ranged from } \\
29 \text { to } 34 \\
\text { years }\end{array}$ & $\begin{array}{l}\text { Parents } \\
\text { living in } \\
\text { downtown } \\
\text { Shantou, } \\
\text { with a child } \\
\text { under the } \\
\text { age of } 6 \\
\text { years living } \\
\text { in the same } \\
\text { household, } \\
\text { and who } \\
\text { had been a } \\
\text { primary } \\
\text { driver and } \\
\text { driving } \\
\text { more than } \\
\text { one time } \\
\text { every week } \\
\text { for at least } \\
2 \text { years. }\end{array}$ & $\begin{array}{l}\text { Perceptions } \\
\text { and } \\
\text { experiences } \\
\text { regarding } \\
\text { use of child } \\
\text { safety } \\
\text { restraint. }\end{array}$ & $\begin{array}{l}20 \\
\text { participants } \\
\text { who met the } \\
\text { selection } \\
\text { from the } \\
1069 \text { drivers } \\
\text { who had } \\
\text { previously } \\
\text { participated } \\
\text { in an } \\
\text { observational } \\
\text { survey, and } \\
\text { expressed } \\
\text { willingness to } \\
\text { be contacted } \\
\text { for future } \\
\text { study were } \\
\text { identified for } \\
\text { IDIs. A small } \\
\text { gift of child } \\
\text { stationery } \\
\text { and a toy } \\
\text { were given. }\end{array}$ & $\begin{array}{l}\text { Face-to-face } \\
\text { interviews } \\
\text { were } \\
\text { conducted } \\
\text { by one } \\
\text { trained } \\
\text { author. Each } \\
\text { of the } \\
\text { interviews } \\
\text { lasted } 35 \text { to } \\
50 \text { minutes } \\
\text { and was } \\
\text { guided by a } \\
\text { topic guide } \\
\text { designed } \\
\text { based on the } \\
\text { Health Belief } \\
\text { Model. All of } \\
\text { questions } \\
\text { were open- } \\
\text { ended. }\end{array}$ & $\begin{array}{l}\text { Research } \\
\text { team } \\
\text { familiarise } \\
\text { d itself } \\
\text { with the } \\
\text { data and } \\
\text { to identify } \\
\text { patterns } \\
\text { and their } \\
\text { links to the } \\
\text { theoretical } \\
\text { framework } \\
\text {. Thematic } \\
\text { analyses } \\
\text { were done } \\
\text { using } \\
\text { NVivo } 10 . \\
\text { Finally, the } \\
\text { bilingual } \\
\text { and } \\
\text { bicultural } \\
\text { research } \\
\text { team } \\
\text { evaluated } \\
\text { the data } \\
\text { and } \\
\text { results. }\end{array}$ \\
\hline
\end{tabular}




\begin{tabular}{|c|c|c|c|c|c|c|c|}
\hline $\begin{array}{l}\text { Nelson } \\
201535 \\
\text { (Saudi } \\
\text { Arabia) }\end{array}$ & $\begin{array}{l}\text { To identify } \\
\text { the salient } \\
\text { beliefs of } \\
\text { pregnant } \\
\text { women in } \\
\text { Saudi } \\
\text { Arabia } \\
\text { that may } \\
\text { influence } \\
\text { their } \\
\text { intentions } \\
\text { to use } \\
\text { infant } \\
\text { restraints } \\
\text { for their } \\
\text { children }\end{array}$ & FGDs. & $\begin{array}{l}25 \\
\text { pregnant } \\
\text { women } \\
\text { participate } \\
\text { d in } 2 \text { FGDs. }\end{array}$ & $\begin{array}{l}\text { Behavioural } \\
\text { ' } \\
\text { and control } \\
\text { beliefs } \\
\text { were } \\
\text { elicited. }{ }^{36}\end{array}$ & $\begin{array}{l}\text { Pregnant } \\
\text { women from } \\
\text { Dallah } \\
\text { Hospital } \\
\text { were eligible } \\
\text { to participate } \\
\text { regardless of } \\
\text { the number } \\
\text { of children. } \\
\text { The } \\
\text { hospital's } \\
\text { representativ } \\
\text { es } \\
\text { approached } \\
\text { individuals } \\
\text { during their } \\
\text { regular } \\
\text { prenatal } \\
\text { appointment } \\
\text { s with an } \\
\text { invitation to } \\
\text { participate in } \\
\text { the study } \\
\text { and } \\
\text { explained the } \\
\text { requirements } \\
\text {. Informed } \\
\text { consent was } \\
\text { obtained } \\
\text { prior to the } \\
\text { start of each } \\
\text { of the FGDs. } \\
\text { participants } \\
\text { received a } \\
\text { gift card } \\
\text { equivalent to } \\
\$ 13.33 .\end{array}$ & $\begin{array}{l}\text { The FGDs } \\
\text { were } \\
\text { moderated } \\
\text { by the health } \\
\text { educator } \\
\text { from the } \\
\text { research } \\
\text { team and } \\
\text { were digitally } \\
\text { recorded, } \\
\text { after which } \\
\text { they were } \\
\text { transcribed } \\
\text { and } \\
\text { translated } \\
\text { into English } \\
\text { by an } \\
\text { independent } \\
\text { translator. A } \\
\text { topic guide } \\
\text { was used. }\end{array}$ & $\begin{array}{l}\text { Researcher } \\
\text { s used the } \\
\text { cut-and- } \\
\text { paste } \\
\text { technique, } \\
\text { where the } \\
\text { transcript } \\
\text { of the } \\
\text { discussions } \\
\text { was cut up } \\
\text { into } \\
\text { segments } \\
\text { and } \\
\text { grouped } \\
\text { into } \\
\text { individual } \\
\text { constructs. } \\
\text { Statements } \\
\text { and } \\
\text { comments } \\
\text { of each } \\
\text { individual } \\
\text { section } \\
\text { (behaviour } \\
\text { al, } \\
\text { normative, } \\
\text { and } \\
\text { control } \\
\text { belief) } \\
\text { were then } \\
\text { analysed } \\
\text { and sorted } \\
\text { into } \\
\text { specific } \\
\text { logical } \\
\text { categories. } \\
\text { Each } \\
\text { category } \\
\text { was then } \\
\text { analysed } \\
\text { and a } \\
\text { belief } \\
\text { statement } \\
\text { was } \\
\text { formulated }\end{array}$ \\
\hline
\end{tabular}




\begin{tabular}{|c|c|c|c|c|c|c|c|}
\hline $\begin{array}{l}\text { Liu } 201637 \\
\text { (China) }\end{array}$ & $\begin{array}{l}\text { To } \\
\text { investigat } \\
\text { e the } \\
\text { knowledg } \\
\text { e, } \\
\text { attitudes, } \\
\text { and } \\
\text { intended } \\
\text { behaviour } \\
\text { s of child } \\
\text { safety } \\
\text { seat use } \\
\text { among } \\
\text { parents of } \\
\text { newborns } \\
\text { and to } \\
\text { explore } \\
\text { expectant } \\
\text { mothers' } \\
\text { views and } \\
\text { decisions } \\
\text { regarding } \\
\text { child } \\
\text { safety } \\
\text { seat use. }\end{array}$ & $\begin{array}{l}\text { Mixed } \\
\text { method- } \\
\text { cross- } \\
\text { sectional } \\
\text { survey and } \\
\text { semi- } \\
\text { structured } \\
\text { interview. }\end{array}$ & $\begin{array}{l}\text { The } \\
\text { inclusion } \\
\text { criteria } \\
\text { were: >12 } \\
\text { weeks } \\
\text { pregnant; } \\
\text { owned a } \\
\text { car; and } \\
\text { agreed to } \\
\text { participate } \\
\text { through } \\
\text { signed } \\
\text { consent. } \\
\text { Those with } \\
\text { high risk } \\
\text { pregnancy } \\
\text { or who } \\
\text { were } \\
\text { unable to } \\
\text { complete } \\
\text { more than } \\
15 \text { minutes } \\
\text { of an in- } \\
\text { person } \\
\text { interview } \\
\text { were } \\
\text { excluded. } \\
\text { The } \\
\text { targeted } \\
\text { sample size } \\
\text { was } 30 \text { to } \\
\text { reach } \\
\text { saturation. }\end{array}$ & $\begin{array}{l}\text { Views and } \\
\text { decisions } \\
\text { regarding } \\
\text { child safety } \\
\text { seat use. }\end{array}$ & $\begin{array}{l}\text { Pregnant } \\
\text { women who } \\
\text { sought } \\
\text { prenatal care } \\
\text { at Shantou } \\
\text { Women's and } \\
\text { Children's } \\
\text { Hospital } \\
\text { were } \\
\text { recruited for } \\
\text { a semi- } \\
\text { structured } \\
\text { interview. } \\
\text { Nothing else } \\
\text { reported. } \\
\text { The study } \\
\text { protocol, } \\
\text { along with } \\
\text { the consent } \\
\text { process, was } \\
\text { approved by } \\
\text { Medical } \\
\text { Ethics } \\
\text { Committees } \\
\text { of Shantou } \\
\text { University } \\
\text { Medical } \\
\text { College. }\end{array}$ & $\begin{array}{l}\text { A semi- } \\
\text { structured } \\
\text { interview } \\
\text { guide was } \\
\text { used. } \\
\text { Interviews } \\
\text { were } \\
\text { conducted in } \\
\text { a private } \\
\text { room in the } \\
\text { hospital and } \\
\text { lasted about } \\
\text { 30 minutes. } \\
\text { All interviews } \\
\text { were audio } \\
\text { recorded and } \\
\text { transcribed } \\
\text { into } \\
\text { electronic } \\
\text { documents } \\
\text { word-for- } \\
\text { word on the } \\
\text { same day by } \\
\text { another } \\
\text { author of } \\
\text { this } \\
\text { manuscript } \\
\text { before data } \\
\text { analysis. }\end{array}$ & $\begin{array}{l}\text { The } \\
\text { transcribed } \\
\text { electronic } \\
\text { documents } \\
\text { were } \\
\text { imported } \\
\text { into NVivo } \\
8.0 \text { and } \\
\text { read } \\
\text { repeatedly } \\
\text { by two } \\
\text { researcher } \\
\text { s, who } \\
\text { coded and } \\
\text { analysed } \\
\text { them } \\
\text { thematicall } \\
\text { y. The data } \\
\text { and results } \\
\text { were } \\
\text { assessed } \\
\text { by } \\
\text { researcher } \\
\text { s who } \\
\text { speak } \\
\text { English, } \\
\text { Chaozhou- } \\
\text { Shantou } \\
\text { dialectal } \\
\text { and } \\
\text { Mandarin, } \\
\text { the } \\
\text { languages } \\
\text { used by } \\
\text { the } \\
\text { participant } \\
\text { s. }\end{array}$ \\
\hline
\end{tabular}




\begin{tabular}{|c|c|c|c|c|c|c|c|}
\hline $\begin{array}{l}\text { Fleisher } \\
2017^{38} \\
\text { (USA) }\end{array}$ & $\begin{array}{l}\text { To assess } \\
\text { perspectiv } \\
\text { es on and } \\
\text { use of } \\
\text { existing } \\
\text { tools to } \\
\text { guide car } \\
\text { seat } \\
\text { installatio } \\
\mathrm{n} \text {, as well } \\
\text { as to } \\
\text { explore } \\
\text { the } \\
\text { acceptabil } \\
\text { ity of } \\
\text { using a } \\
\text { mobile } \\
\text { app to } \\
\text { guide car } \\
\text { seat } \\
\text { installatio } \\
\text { n. }\end{array}$ & $\begin{array}{l}\text { Mixed } \\
\text { methods } \\
\text { study } \\
\text { consisting of } \\
\text { survey, and } \\
\text { FGDs using } \\
\text { phenomenol } \\
\text { ogical } \\
\text { approach. }\end{array}$ & $\begin{array}{l}\text { Parent/ } \\
\text { guardian } \\
\text { with a child } \\
\text { between } \\
\text { ages } 0 \text { and } \\
5 \text { years } \\
\text { (inclusive) } \\
\text { treated at } \\
\text { the } \\
\text { participatin } \\
\text { g practice. } \\
\text { Participant } \\
\text { eligibility } \\
\text { was } \\
\text { determined } \\
\text { according } \\
\text { to the age } \\
\text { of the child, } \\
\text { child race, } \\
\text { child } \\
\text { medical } \\
\text { insurance } \\
\text { type } \\
\text { (private or } \\
\text { Medicaid), } \\
\text { parent } \\
\text { report of } \\
\text { transportin } \\
\text { g a child in } \\
\text { a car seat } \\
\text { at least } 2 \\
\text { times per } \\
\text { week, } \\
\text { parent } \\
\text { report of } \\
\text { installing } \\
\text { any type of } \\
\text { car seat at } \\
\text { least } 5 \\
\text { times in the } \\
\text { last } 6 \\
\text { months, } \\
\text { and parent } \\
\text { ability to } \\
\text { travel to } \\
\text { the local } \\
\text { paediatric } \\
\text { practice. }\end{array}$ & $\begin{array}{l}\text { Perspective } \\
\mathrm{s} \text { and } \\
\text { experiences } \\
\text {. }\end{array}$ & $\begin{array}{l}\text { Primary care } \\
\text { practices } \\
\text { were chosen } \\
\text { based on } \\
\text { geographic } \\
\text { location to } \\
\text { ensure } \\
\text { variability in } \\
\text { participant } \\
\text { race/ } \\
\text { ethnicity, } \\
\text { community } \\
\text { setting and } \\
\text { socioeconom } \\
\text { ic status. A } \\
\text { list of } 400 \\
\text { potentially } \\
\text { eligible } \\
\text { participants } \\
\text { was } \\
\text { generated for } \\
\text { each } \\
\text { practice. } \\
\text { Eligible } \\
\text { parents/ } \\
\text { caregivers } \\
\text { were invited } \\
\text { to participate } \\
\text { by mail and } \\
\text { telephone. } \\
\text { Parents and } \\
\text { caregivers } \\
\text { who } \\
\text { contacted or } \\
\text { were } \\
\text { contacted by } \\
\text { study staff } \\
\text { and screened } \\
\text { to be eligible } \\
\text { were } \\
\text { enrolled and } \\
\text { scheduled for } \\
\text { a focus group } \\
\text { until an initial } \\
\text { enrolment } \\
\text { goal of at } \\
\text { least } 40 \\
\text { participants } \\
\text { was } \\
\text { arhioved }\end{array}$ & $\begin{array}{l}\text { The lead } \\
\text { author who } \\
\text { has training } \\
\text { and } \\
\text { extensive } \\
\text { experience in } \\
\text { focus group } \\
\text { facilitation } \\
\text { led the FGDs. } \\
\text { The } \\
\text { participants } \\
\text { were } \\
\text { recruited } \\
\text { through each } \\
\text { primary care } \\
\text { practice and } \\
\text { were not } \\
\text { familiar with } \\
\text { the research } \\
\text { team. The } \\
\text { FGDs were } \\
\text { conducted at } \\
\text { each of the } \\
\text { participating } \\
\text { clinics and } \\
\text { therefore } \\
\text { convenient } \\
\text { for } \\
\text { participants. } \\
\text { A } \\
\text { phenomenol } \\
\text { ogical } \\
\text { research } \\
\text { approach } \\
\text { was } \\
\text { employed. }\end{array}$ & $\begin{array}{l}\text { FGD } \\
\text { recordings } \\
\text { were } \\
\text { transcribed } \\
\text { and } \\
\text { analysed } \\
\text { using } \\
\text { inductive } \\
\text { thematic } \\
\text { analysis to } \\
\text { identify } \\
\text { patterns } \\
\text { across the } \\
\text { dataset. } \\
\text { Three } \\
\text { reviewers } \\
\text { conducted } \\
\text { initial } \\
\text { coding and } \\
\text { then } \\
\text { coding } \\
\text { conference } \\
\text { s were } \\
\text { held to } \\
\text { discuss } \\
\text { and } \\
\text { resolve } \\
\text { discrepanci } \\
\text { es. In the } \\
\text { first phase } \\
\text { of analysis, } \\
\text { apparent } \\
\text { themes } \\
\text { were } \\
\text { identified } \\
\text { from an } \\
\text { initial } \\
\text { review of } \\
\text { the data. } \\
\text { FGD } \\
\text { recordings } \\
\text { were then } \\
\text { transcribed } \\
\text { and read in } \\
\text { entirety by } \\
\text { members } \\
\text { of the } \\
\text { research } \\
\text { team, who } \\
\text { identified }\end{array}$ \\
\hline
\end{tabular}




\begin{tabular}{|c|c|c|c|c|c|c|c|}
\hline $\begin{array}{l}\text { Hunter } \\
201739 \\
\text { (Australia) }\end{array}$ & $\begin{array}{l}\text { To explore } \\
\text { parental } \\
\text { knowledg } \\
\text { e and } \\
\text { attitudes } \\
\text { and self- } \\
\text { reported } \\
\text { child car } \\
\text { seat use. }\end{array}$ & $\begin{array}{l}\text { Mixed } \\
\text { methods } \\
\text { study } \\
\text { consisting of } \\
\text { survey, and } \\
\text { FGDs using } \\
\text { the } \\
\text { PRECEDE- } \\
\text { PROCEED } \\
\text { conceptual } \\
\text { framework. }\end{array}$ & $\begin{array}{l}\text { Parents or } \\
\text { carers } \\
\text { whose } \\
\text { children } \\
\text { aged } 3-5 \\
\text { years were } \\
\text { enrolled in } \\
\text { any of the } \\
\text { three early } \\
\text { learning } \\
\text { centres } \\
\text { (two } \\
\text { preschools } \\
\text { and one } \\
\text { long day } \\
\text { care } \\
\text { centre) in } \\
\text { regional } \\
\text { NSW. } \\
10 \text { in } 3 \\
\text { FGDs. }\end{array}$ & $\begin{array}{l}\text { Knowledge } \\
\text { and } \\
\text { attitudes. }\end{array}$ & $\begin{array}{l}\text { Participants } \\
\text { were } \\
\text { recruited } \\
\text { through } \\
\text { posters that } \\
\text { were } \\
\text { displayed at } \\
\text { the centres. } \\
\text { The centre } \\
\text { director was } \\
\text { also asked to } \\
\text { encourage } \\
\text { parents of } \\
\text { Aboriginal } \\
\text { children to } \\
\text { attend and } \\
\text { directly } \\
\text { approached } \\
\text { participants } \\
\text { through } \\
\text { personal } \\
\text { invitation. }\end{array}$ & $\begin{array}{l}\text { A researcher } \\
\text { experienced } \\
\text { in qualitative } \\
\text { methods } \\
\text { conducted } \\
\text { three FGDs } \\
\text { of } 60-90 \text { min } \\
\text { duration at } \\
\text { each of the } \\
\text { centres, 6- } \\
14 \text { weeks } \\
\text { after the } \\
\text { surveys were } \\
\text { administered } \\
\text {. Focus } \\
\text { groups were } \\
\text { conducted } \\
\text { during the } \\
\text { day at times } \\
\text { convenient } \\
\text { for parents } \\
\text { and centre } \\
\text { staff. }\end{array}$ & $\begin{array}{l}\text { FGD } \\
\text { recordings } \\
\text { were } \\
\text { listened to } \\
\text { in full then } \\
\text { transcribed } \\
\text { verbatim } \\
\text { by a } \\
\text { transcripti } \\
\text { on service } \\
\text { and then } \\
\text { checked } \\
\text { for } \\
\text { accuracy } \\
\text { by the first } \\
\text { author. } \\
\text { Each } \\
\text { transcripti } \\
\text { on was } \\
\text { read and } \\
\text { re-read } \\
\text { and codes } \\
\text { were } \\
\text { developed } \\
\text { deductively } \\
\text {, based } \\
\text { upon the } \\
\text { theoretical } \\
\text { framework } \\
. \text { Non- } \\
\text { verbal } \\
\text { communic } \\
\text { ation was } \\
\text { not } \\
\text { systematic } \\
\text { ally } \\
\text { interpreted } \\
\text {. NVIVO } 9 \\
\text { to organise } \\
\text { the } \\
\text { qualitative } \\
\text { data. }\end{array}$ \\
\hline
\end{tabular}




\begin{tabular}{|c|c|c|c|c|c|c|c|}
\hline $\begin{array}{l}\text { Mckenzie } \\
201740 \\
\text { (USA) }\end{array}$ & $\begin{array}{l}\text { To } \\
\text { determine } \\
\text { prevalenc } \\
\text { e of child } \\
\text { safety } \\
\text { seat use, } \\
\text { awarenes } \\
\text { s of and } \\
\text { barriers to } \\
\text { use; and } \\
\text { to use the } \\
\text { informatio } \\
\text { n gained } \\
\text { in the first } \\
\text { phase to } \\
\text { develop, } \\
\text { implemen } \\
\text { t, and } \\
\text { evaluate a } \\
\text { child } \\
\text { passenger } \\
\text { safety } \\
\text { program } \\
\text { for Somali } \\
\text { families } \\
\text { living in } \\
\text { Columbus } \\
\text {, Ohio } \\
\text { that is } \\
\text { both } \\
\text { sustainabl } \\
\text { e and } \\
\text { culturally } \\
\text { appropria } \\
\text { te. }\end{array}$ & $\begin{array}{l}\text { Mixed } \\
\text { methods } \\
\text { study } \\
\text { consisting of } \\
\text { survey, IDIs } \\
\text { and } 2 \text { FGDs } \\
\text { to develop } \\
\text { an } \\
\text { intervention } \\
\text { followed by a } \\
\text { telephonic } \\
\text { survey to get } \\
\text { feedback on } \\
\text { the } \\
\text { intervention. } \\
\text { The } \\
\text { intervention } \\
\text { was a video } \\
\text { which was } \\
\text { given to } \\
\text { community } \\
\text { leaders to } \\
\text { show - this } \\
\text { was } \\
\text { developed } \\
\text { based on } \\
\text { results of a } \\
\text { component } \\
\text { of this study. }\end{array}$ & $\begin{array}{l}\text { Key } \\
\text { informants } \\
(10) \text { in the } \\
\text { local Somali } \\
\text { community } \\
\text { were } \\
\text { initially } \\
\text { identified } \\
\text { by the } \\
\text { executive } \\
\text { director of } \\
\text { a Somali } \\
\text { family } \\
\text { alliance } \\
\text { organizatio } \\
\mathrm{n} \text { in } \\
\text { Columbus, } \\
\text { Ohio. } \\
\text { Additional } \\
\text { community } \\
\text { leaders } \\
\text { were } \\
\text { identified } \\
\text { as the } \\
\text { interviews } \\
\text { progressed. } \\
\text { To } \\
\text { participate } \\
\text { in FGDs, } \\
\text { parents had } \\
\text { to be } \\
\text { Somali, at } \\
\text { least } 18 \\
\text { years of } \\
\text { age, and } \\
\text { have at } \\
\text { least one } \\
\text { child } \leq 8 \\
\text { years old. }\end{array}$ & $\begin{array}{l}\text { Knowledge } \\
\text { of and } \\
\text { barriers to } \\
\text { proper use } \\
\text { in order to } \\
\text { inform } \\
\text { developme } \\
\text { nt, } \\
\text { implement } \\
\text { ation, and } \\
\text { initial } \\
\text { evaluation } \\
\text { of a } \\
\text { culturally } \\
\text { appropriate } \\
\text { interventio } \\
\mathrm{n} \text { for Somali } \\
\text { families. }\end{array}$ & $\begin{array}{l}\text { Key } \\
\text { informant } \\
(\mathrm{n}=10) \text { were } \\
\text { initially } \\
\text { identified by } \\
\text { the executive } \\
\text { director of a } \\
\text { Somali family } \\
\text { alliance } \\
\text { organisation } \\
\text { and later } \\
\text { snowballing } \\
\text { done. No } \\
\text { information } \\
\text { is provided } \\
\text { on how } \\
\text { Somali } \\
\text { parents } \\
\text { (n=30) were } \\
\text { recruited. } \\
\text { The study } \\
\text { was } \\
\text { approved by } \\
\text { the } \\
\text { Institutional } \\
\text { Review } \\
\text { Board at the } \\
\text { Research } \\
\text { Institute at } \\
\text { Nationwide } \\
\text { Children's } \\
\text { Hospital. }\end{array}$ & $\begin{array}{l}\text { No } \\
\text { information } \\
\text { provided on } \\
\text { data } \\
\text { collection of } \\
\text { key } \\
\text { informants, } \\
\text { but for FGD } \\
\text { it is } \\
\text { mentioned } \\
\text { that an } \\
\text { interpreter } \\
\text { was used, } \\
\text { and open- } \\
\text { ended } \\
\text { questions } \\
\text { were asked. }\end{array}$ & $\begin{array}{l}\text { Only } \\
\text { mentions } \\
\text { that the } \\
\text { data was } \\
\text { recorded } \\
\text { and } \\
\text { transcribed } \\
\text { and } \\
\text { common } \\
\text { themes } \\
\text { were } \\
\text { extracted } \\
\text { upon } \\
\text { review and } \\
\text { briefly } \\
\text { summarize } \\
\text { d. }\end{array}$ \\
\hline
\end{tabular}




\begin{tabular}{|c|c|c|c|c|c|c|c|}
\hline $\begin{array}{l}\text { Hall } 201841 \\
\text { (Australia) }\end{array}$ & $\begin{array}{l}\text { To explore } \\
\text { how child } \\
\text { restraint } \\
\text { use might } \\
\text { function } \\
\text { as part of } \\
\text { an } \\
\text { individual' } \\
\text { s } \\
\text { capability, } \\
\text { opportuni } \\
\text { ty, and } \\
\text { motivatio } \\
\mathrm{n} \text { and } \\
\text { how this } \\
\text { varies } \\
\text { across } \\
\text { high } \\
\text { education } \\
\text { and } \\
\text { income } \\
\text { (high SES), } \\
\text { low } \\
\text { education } \\
\text { and } \\
\text { income } \\
\text { (low SES), } \\
\text { and } \\
\text { culturally } \\
\text { and } \\
\text { linguistical } \\
\text { ly diverse } \\
\text { (CALD) } \\
\text { users }\end{array}$ & FGDs. & $\begin{array}{l}\text { All parents } \\
\text { or carers } \\
\text { over } 18 \\
\text { years and } \\
\text { conversant } \\
\text { in English } \\
\text { were } \\
\text { eligible. } \\
\text { Two groups } \\
\text { each of } \\
\text { high SES } \\
\text { participants } \\
\text {, CALD } \\
\text { participants } \\
\text {, and low } \\
\text { SES } \\
\text { participants } \\
\text {. All } \\
\text { participants } \\
\text { had some } \\
\text { previous } \\
\text { experience } \\
\text { in installing } \\
\text { a child } \\
\text { restraint } \\
\text { system or } \\
\text { securing a } \\
\text { child into a } \\
\text { restraint. } \\
\text { Participants } \\
\text { were } \\
\text { reimbursed } \\
\text { AUD\$25.00 } \\
\text { for their } \\
\text { travel costs. }\end{array}$ & $\begin{array}{l}\text { Perceptions } \\
\text { (insights) } \\
\text { and } \\
\text { experiences } \\
\text {. }\end{array}$ & $\begin{array}{l}\text { High SES } \\
\text { participants } \\
\text { were } \\
\text { recruited } \\
\text { through } \\
\text { university } \\
\text { and research } \\
\text { organisation } \\
\text { email } \\
\text { distribution } \\
\text { channels. } \\
\text { Community } \\
\text { playgroups } \\
\text { specifically } \\
\text { for English as } \\
\text { second } \\
\text { language } \\
\text { residents in } \\
\text { South- } \\
\text { eastern } \\
\text { Sydney, and } \\
\text { community } \\
\text { playgroups in } \\
\text { areas low SES } \\
\text { as indicated } \\
\text { by the } \\
\text { Australian } \\
\text { Government } \\
\text { were used to } \\
\text { recruit CALD } \\
\text { and low SES } \\
\text { participants. }\end{array}$ & $\begin{array}{l}\text { Two } \\
\text { researchers } \\
\text { attended } \\
\text { each FGD; } \\
\text { one } \\
\text { facilitated } \\
\text { discussion } \\
\text { using a semi- } \\
\text { structured } \\
\text { discussion } \\
\text { guide and } \\
\text { the other } \\
\text { took notes. } \\
\text { The } \\
\text { discussion } \\
\text { guide was } \\
\text { formulated } \\
\text { on a review } \\
\text { of factors } \\
\text { previously } \\
\text { reported to } \\
\text { be } \\
\text { associated } \\
\text { with errors in } \\
\text { child } \\
\text { restraint use. }\end{array}$ & $\begin{array}{l}\text { The COM- } \\
\text { B model of } \\
\text { behaviour } \\
\text { was used } \\
\text { to } \\
\text { deductivel } \\
\text { y analyse } \\
\text { discussions } \\
\text { Transcripts } \\
\text { and } \\
\text { discussion } \\
\text { notes were } \\
\text { then coded } \\
\text { according } \\
\text { to these } \\
\text { categories } \\
\text { independe } \\
\text { ntly by two } \\
\text { researcher } \\
\text { s using } \\
\text { QSR } \\
\text { Internation } \\
\text { al's NVivo } \\
11 \\
\text { Software. } \\
\text { This } \\
\text { included } \\
\text { any } \\
\text { mention of } \\
\text { any issue } \\
\text { relevant to } \\
\text { these pre- } \\
\text { defined } \\
\text { categories. } \\
\text { Where any } \\
\text { consistenci } \\
\text { es } \\
\text { between } \\
\text { researcher } \\
\text { s occurred, } \\
\text { these were } \\
\text { discussed } \\
\text { between } \\
\text { the } \\
\text { researcher } \\
\text { s until } \\
\text { consensus } \\
\text { was } \\
\text { rearherd }\end{array}$ \\
\hline
\end{tabular}



Focus of included studies

Most of the studies intended to understand perceptions, attitudes, and barriers to the use of child restraints in motor vehicles. The qualitative studies were standalone projects except for three studies, which were undertaken in conjunction with an intervention 293138.

\section{Type of studies}

The studies included were either standalone qualitative methodologies or mixed methods. The different types of studies included were:

- focus group discussions only 21 24-26 28-32 3541

- in-depth interviews only ${ }^{34}$

- focus groups and in-depth interviews 23

- mixed method study designs 37-40

Study participants

All but two of the included studies targeted parents or caregivers of children as participants. Only one study 23 included children in a qualitative component in addition to parents or caregivers. One study included known violators of the child restraints law, including parents and caregivers 25 and two studies focussed on pregnant women 35 37. Many studies included participants from culturally and linguistically diverse minorities 243240 - two looked specifically at low-income communities ${ }^{3041}$.

Study setting

All the qualitative studies included were from high-income (USA=9, Australia =4, Saudi Arabia =1) $(H I C)$ or upper-middle-income countries (China=3) (UMIC). Studies were mostly community-based.

Methodological quality of the included studies

The methodological quality of the included studies as assessed by the CASP tool is summarised in Table 2. Most studies were clear about their research aims, had justified the use of a qualitative design and the overall design was appropriate. The recruitment strategy was not clear in four studies 21283537 . Nine studies 212425283032353940 were adjudged to not have sufficiently rigorous data analysis methods (and one study was adjudged unclear ${ }^{23}$ on several counts including for methods of analysis), reflexivity not being reported and data saturation not being reached or not reported. Three studies 212531 were adjudged to not have clear statement of findings since they did not present quotes of the participants to back-up their results and/or did not describe basic characteristics of participants. 
Table 2 : Methodological limitations of included studies using the CASP qualitative study tool 


\begin{tabular}{|c|c|c|c|c|c|c|c|c|c|c|}
\hline $\begin{array}{c}\text { CASP } \\
\text { criteri } \\
\text { a }^{18}\end{array}$ & $\begin{array}{l}\text { Was } \\
\text { ther } \\
\text { e a } \\
\text { clear } \\
\text { state } \\
\text { men } \\
\text { t of } \\
\text { the } \\
\text { aims } \\
\text { of } \\
\text { the } \\
\text { rese } \\
\text { arch } \\
\text { ? }\end{array}$ & $\begin{array}{l}\text { Is a } \\
\text { qu } \\
\text { alit } \\
\text { ati } \\
\text { ve } \\
\text { me } \\
\text { th } \\
\text { od } \\
\text { olo } \\
\text { gy } \\
\text { ap } \\
\text { pr } \\
\text { op } \\
\text { ria } \\
\text { te? }\end{array}$ & $\begin{array}{l}\text { Was } \\
\text { the } \\
\text { resea } \\
\text { rch } \\
\text { desig } \\
n \\
\text { appr } \\
\text { opria } \\
\text { te to } \\
\text { addr } \\
\text { ess } \\
\text { the } \\
\text { aims } \\
\text { of } \\
\text { the } \\
\text { resea } \\
\text { rch? }\end{array}$ & $\begin{array}{l}\text { Was } \\
\text { the } \\
\text { recru } \\
\text { itme } \\
\text { nt } \\
\text { strat } \\
\text { egy } \\
\text { appr } \\
\text { opria } \\
\text { te to } \\
\text { the } \\
\text { aims } \\
\text { of } \\
\text { the } \\
\text { resea } \\
\text { rch? }\end{array}$ & $\begin{array}{l}\text { Was } \\
\text { the } \\
\text { data } \\
\text { colle } \\
\text { cted } \\
\text { in a } \\
\text { way } \\
\text { that } \\
\text { addr } \\
\text { esse } \\
\text { d the } \\
\text { resea } \\
\text { rch } \\
\text { issue } \\
?\end{array}$ & $\begin{array}{l}\text { Has } \\
\text { the } \\
\text { relati } \\
\text { onshi } \\
\text { p } \\
\text { betwe } \\
\text { en } \\
\text { resear } \\
\text { cher } \\
\text { and } \\
\text { partici } \\
\text { pants } \\
\text { been } \\
\text { adequ } \\
\text { ately } \\
\text { consid } \\
\text { ered? }\end{array}$ & $\begin{array}{l}\text { Hav } \\
\text { e } \\
\text { ethic } \\
\text { al } \\
\text { issu } \\
\text { es } \\
\text { bee } \\
n \\
\text { take } \\
n \\
\text { into } \\
\text { cons } \\
\text { ider } \\
\text { atio } \\
\text { n? }\end{array}$ & $\begin{array}{l}\text { Was } \\
\text { the } \\
\text { data } \\
\text { analysi } \\
\text { s } \\
\text { sufficie } \\
\text { ntly } \\
\text { rigorou } \\
\text { s? }\end{array}$ & $\begin{array}{l}\text { Is } \\
\text { the } \\
\text { re } \\
\text { a } \\
\text { cle } \\
\text { ar } \\
\text { sta } \\
\text { te } \\
\text { me } \\
\text { nt } \\
\text { of } \\
\text { fin } \\
\text { din } \\
\text { gs? }\end{array}$ & $\begin{array}{l}\text { How valuable is the } \\
\text { research? }\end{array}$ \\
\hline $\begin{array}{l}\text { Rivara } \\
2001 \\
21\end{array}$ & Yes & Yes & Yes & $\begin{array}{l}\text { Uncl } \\
\text { ear }\end{array}$ & No & No & No & No & No & $\begin{array}{l}\text { The study had a small sample } \\
\text { size. Data saturation and } \\
\text { analysis methods were not } \\
\text { properly described. There } \\
\text { was discordance between } \\
\text { the age in objective and } \\
\text { eligibility criteria. Participant } \\
\text { quotes to back up themes } \\
\text { was sparsely presented. }\end{array}$ \\
\hline $\begin{array}{l}\text { Simps } \\
\text { on } \\
2002 \\
23\end{array}$ & Yes & Yes & Yes & Yes & Yes & $\begin{array}{l}\text { Uncle } \\
\text { ar }\end{array}$ & $\begin{array}{l}\text { Uncl } \\
\text { ear }\end{array}$ & $\begin{array}{l}\text { Unclea } \\
r\end{array}$ & Yes & $\begin{array}{l}\text { Not much reported on } \\
\text { analyses to decide on } \\
\text { reliability, validity, or } \\
\text { reflexivity. However, the } \\
\text { results discussed how the } \\
\text { study contributed to } \\
\text { determining parental } \\
\text { differences between optimal } \\
\text { child restraint use and the } \\
\text { need for quantitative studies } \\
\text { in the future. It was not } \\
\text { clear why the ethics exempt } \\
\text { status was given for this } \\
\text { research }\end{array}$ \\
\hline
\end{tabular}




\begin{tabular}{|c|c|c|c|c|c|c|c|c|c|c|}
\hline $\begin{array}{l}\text { Lee } \\
2003 \\
24\end{array}$ & Yes & Yes & Yes & Yes & No & $\begin{array}{l}\text { Uncle } \\
\text { ar }\end{array}$ & Yes & No & Yes & $\begin{array}{l}\text { Data saturation was not } \\
\text { mentioned. Not much detail } \\
\text { about analysis methodology } \\
\text { or results was presented and } \\
\text { only a narrative report was } \\
\text { provided with positionality } \\
\text { and reflexivity remaining } \\
\text { unclear. }\end{array}$ \\
\hline $\begin{array}{l}\text { Agran } \\
2004 \\
25\end{array}$ & Yes & Yes & Yes & Yes & $\begin{array}{l}\text { Uncl } \\
\text { ear }\end{array}$ & No & Yes & No & No & $\begin{array}{l}\text { The study did not include } \\
\text { much detail about data } \\
\text { collection. Reflexivity and } \\
\text { data saturation were not } \\
\text { mentioned. Basic } \\
\text { characteristics of the } \\
\text { participants or quotes were } \\
\text { not reported. However, the } \\
\text { researcher briefly discussed } \\
\text { the contribution the study in } \\
\text { terms of implementation of } \\
\text { laws. }\end{array}$ \\
\hline $\begin{array}{l}\text { Lenno } \\
\text { n } \\
2007 \\
26\end{array}$ & Yes & Yes & Yes & Yes & Yes & Yes & Yes & Yes & Yes & $\begin{array}{l}\text { The study was well } \\
\text { conducted, and the } \\
\text { researcher discussed the } \\
\text { contribution it made in the } \\
\text { larger context and the issue } \\
\text { of generalisability, reliability } \\
\text { and validity. }\end{array}$ \\
\hline
\end{tabular}




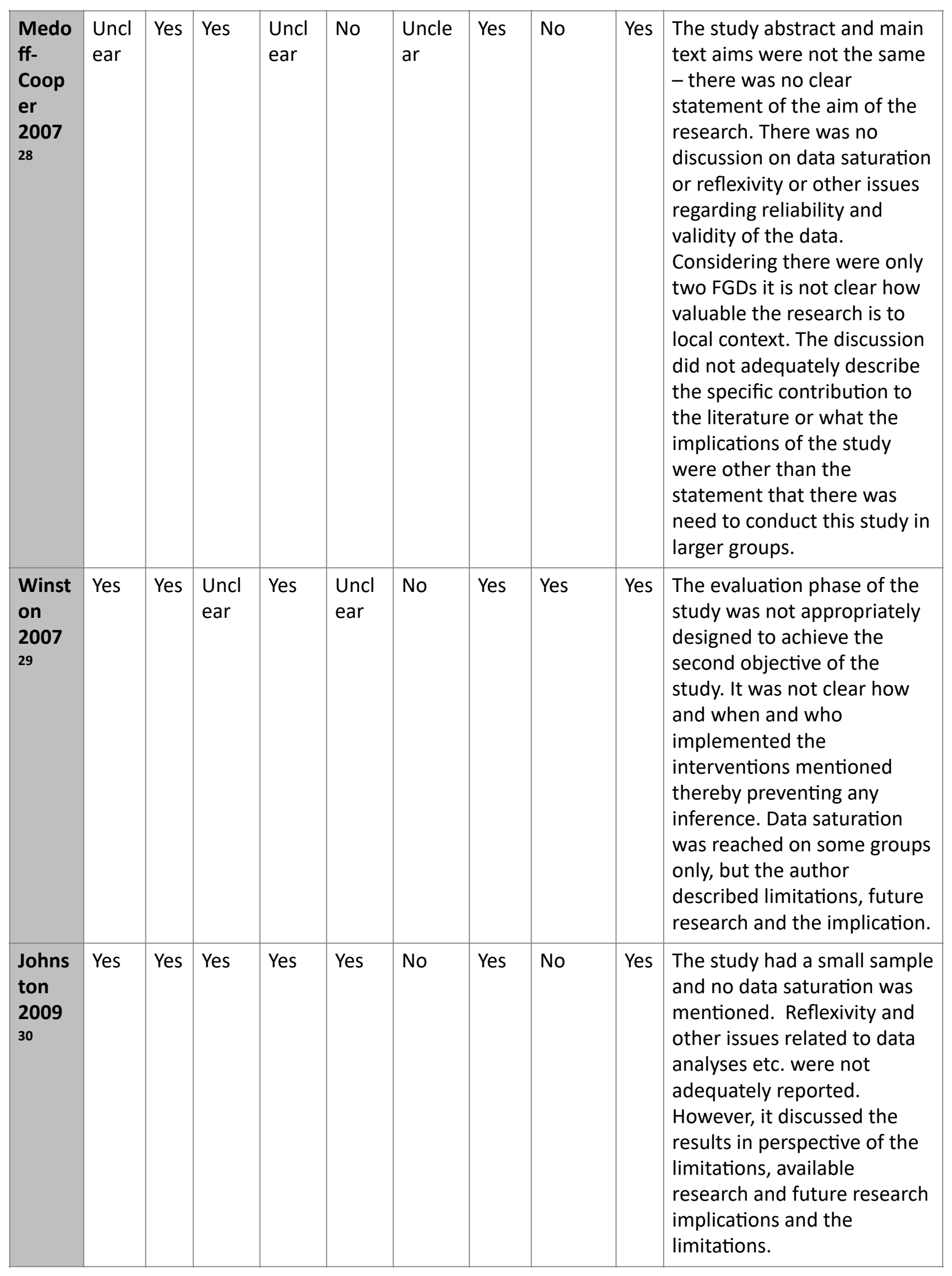




\begin{tabular}{|c|c|c|c|c|c|c|c|c|c|c|}
\hline $\begin{array}{l}\text { Erkob } \\
\text { oni } \\
2010 \\
31\end{array}$ & Yes & Yes & Yes & Yes & No & No & Yes & Yes & No & $\begin{array}{l}\text { The research had a problem } \\
\text { of data saturation and } \\
\text { positionality of researcher } \\
\text { was not described. Results } \\
\text { did not have quotes. The } \\
\text { study, however, noted the } \\
\text { wider literature and the } \\
\text { generalisability in the local } \\
\text { context in comparison to the } \\
\text { original study done in USA } \\
\text { and study limitations. }\end{array}$ \\
\hline $\begin{array}{l}\text { Brow } \\
n \\
2013 \\
32\end{array}$ & Yes & Yes & Yes & Yes & $\begin{array}{l}\text { Uncl } \\
\text { ear }\end{array}$ & $\begin{array}{l}\text { Uncle } \\
\text { ar }\end{array}$ & Yes & No & Yes & $\begin{array}{l}\text { The author identified the } \\
\text { future implication on } \\
\text { research and the } \\
\text { contribution made by the } \\
\text { study. However, the data } \\
\text { were not analysed rigorously } \\
\text { and there was no } \\
\text { information on data } \\
\text { saturation. Not enough } \\
\text { details on reflexivity either. }\end{array}$ \\
\hline $\begin{array}{l}\text { Chen } \\
2014 \\
34\end{array}$ & Yes & Yes & Yes & Yes & Yes & Yes & Yes & Yes & Yes & $\begin{array}{l}\text { The author identified the } \\
\text { future implication on } \\
\text { research and the } \\
\text { contribution made by the } \\
\text { study including the } \\
\text { transferability of the findings } \\
\text { in other settings. Limitations } \\
\text { were discussed. }\end{array}$ \\
\hline $\begin{array}{l}\text { Nelso } \\
n \\
2015 \\
35\end{array}$ & Yes & Yes & Yes & $\begin{array}{l}\text { Uncl } \\
\text { ear }\end{array}$ & Yes & $\begin{array}{l}\text { Uncle } \\
\text { ar }\end{array}$ & Yes & No & Yes & $\begin{array}{l}\text { The researcher did not } \\
\text { clearly state why only } \\
\text { pregnant women were } \\
\text { targeted for the study. Data } \\
\text { saturation, small sample size } \\
\text { and the lack of information } \\
\text { on researcher's role and } \\
\text { reflexivity were limitations. } \\
\text { However, the data did } \\
\text { discuss local implications } \\
\text { considering the larger } \\
\text { literature, as well as } \\
\text { strengths and limitations of } \\
\text { the study. }\end{array}$ \\
\hline
\end{tabular}




\begin{tabular}{|c|c|c|c|c|c|c|c|c|c|c|}
\hline $\begin{array}{l}\text { Liu } \\
2016 \\
37\end{array}$ & Yes & Yes & Yes & $\begin{array}{l}\text { Uncl } \\
\text { ear }\end{array}$ & Yes & No & Yes & Yes & Yes & $\begin{array}{l}\text { The study was well } \\
\text { conducted, and the } \\
\text { researcher discussed the } \\
\text { contribution it made in the } \\
\text { larger context and the issue } \\
\text { of generalisability, reliability } \\
\text { and validity and the need for } \\
\text { future research. However, } \\
\text { there was inadequate } \\
\text { reporting of actual } \\
\text { recruitment strategy and } \\
\text { reflexivity. }\end{array}$ \\
\hline $\begin{array}{l}\text { Fleish } \\
\text { er } \\
2017 \\
38\end{array}$ & Yes & Yes & Yes & Yes & Yes & Yes & Yes & Yes & Yes & $\begin{array}{l}\text { The study was well } \\
\text { conducted, and the } \\
\text { researcher discussed the } \\
\text { contribution it made in the } \\
\text { larger context and the issue } \\
\text { of generalisability, reliability } \\
\text { and validity. }\end{array}$ \\
\hline $\begin{array}{l}\text { Hunte } \\
r \\
2017 \\
39\end{array}$ & Yes & Yes & Yes & Yes & Yes & $\begin{array}{l}\text { Uncle } \\
\text { ar }\end{array}$ & Yes & No & Yes & $\begin{array}{l}\text { The study was well } \\
\text { conducted, and the } \\
\text { researcher discussed the } \\
\text { contribution it made in the } \\
\text { larger context and the issue } \\
\text { of generalisability, reliability } \\
\text { and validity. However, } \\
\text { reflexivity was not reported } \\
\text { and data saturation was not } \\
\text { reached. There was also } \\
\text { some discordance between } \\
\text { the total number of } \\
\text { participants and the } \\
\text { individual participants in } \\
\text { each FGD. }\end{array}$ \\
\hline $\begin{array}{l}\text { McKe } \\
\text { nzie } \\
2017 \\
40\end{array}$ & Yes & Yes & Yes & Yes & No & $\begin{array}{l}\text { Uncle } \\
\text { ar }\end{array}$ & Yes & No & Yes & $\begin{array}{l}\text { The overall design involved } \\
\text { designing an intervention } \\
\text { and obtaining pilot feedback } \\
\text { The design was reasonable } \\
\text { but not much can be said } \\
\text { about the reliability and } \\
\text { validity of the research or } \\
\text { the generalisability of it due } \\
\text { to poor reporting of } \\
\text { methodology and results. } \\
\text { Data saturation and } \\
\text { reflexivity was not } \\
\text { mentioned. }\end{array}$ \\
\hline
\end{tabular}




\begin{tabular}{|l|l|l|l|l|l|l|l|l|l|l|}
\hline $\begin{array}{l}\text { Hall } \\
\mathbf{2 0 1 8} \\
\mathbf{4 1}\end{array}$ & Yes & Yes & Yes & Yes & Yes & $\begin{array}{l}\text { Uncle } \\
\text { ar }\end{array}$ & Yes & Yes & Yes & $\begin{array}{l}\text { The study was well } \\
\text { conducted, and the } \\
\text { researcher discussed the } \\
\text { contribution it made in the } \\
\text { larger context and the issue } \\
\text { of generalisability, reliability } \\
\text { and validity. }\end{array}$ \\
\hline
\end{tabular}




\section{Equity lens using PROGRESS Plus}

We applied an equity lens on a post-hoc basis using the PROGRESS Plus framework to better understand the equity issues around facilitators and barriers for child restraint use ${ }^{17}$ and this is shown in Table 3. However, for many studies the reporting was not detailed enough to enable a better understanding of equity. Almost all the studies were conducted in big cities in high-income countries and seven studies specifically focussed on culturally and linguistically diverse groups 243032 38-41. Studies typically did not intend to explore differences between mothers and fathers. Occupation, a key factor which might influence risk perception and risk-taking behaviour as well as socio-economic status was not reported in eleven studies 21 23-263032 34353738 . Religion was mentioned only in one study 35; socio-economic conditions were not reported in six studies 23-25313235. Social capital was reported only in one study 40. Even when they have reported equity factors, studies did not look specifically on its effect, except when they looked at culturally and linguistically diverse communities. 
Table 3: Equity characteristics of participants in included studies using the PROGRESS Plus framework 


\begin{tabular}{|c|c|c|c|c|c|c|c|c|c|}
\hline Study & $\begin{array}{l}\text { Place of } \\
\text { residenc } \\
\mathrm{e}\end{array}$ & $\begin{array}{l}\text { Race/ } \\
\text { ethnicity/ } \\
\text { culture/ } \\
\text { language }\end{array}$ & $\begin{array}{l}\text { Occupa } \\
\text { tion }\end{array}$ & $\begin{array}{l}\text { Gend } \\
\text { er/ } \\
\text { sex }\end{array}$ & $\begin{array}{l}\text { Religio } \\
n\end{array}$ & $\begin{array}{l}\text { Educati } \\
\text { on }\end{array}$ & $\begin{array}{l}\text { Socio- } \\
\text { economic } \\
\text { Status }\end{array}$ & $\begin{array}{l}\text { Social } \\
\text { capital }\end{array}$ & Plus \\
\hline $\begin{array}{l}\text { Rivara } \\
200121\end{array}$ & $\begin{array}{l}\text { Seattle, } \\
\text { USA }\end{array}$ & $\begin{array}{l}\text { Only } \\
\text { English } \\
\text { speaking } \\
\text { were } \\
\text { included }\end{array}$ & $\begin{array}{l}\text { Not } \\
\text { reporte } \\
\text { d }\end{array}$ & $\begin{array}{l}\text { All } \\
\text { but } \\
\text { three } \\
\text { were } \\
\text { wome } \\
n\end{array}$ & $\begin{array}{l}\text { Not } \\
\text { reporte } \\
\text { d }\end{array}$ & $\begin{array}{l}\text { Not } \\
\text { reporte } \\
\text { d }\end{array}$ & $\begin{array}{l}\text { Conducted in } \\
\text { three } \\
\text { different } \\
\text { socioeconom } \\
\text { ic areas of } \\
\text { the Seattle } \\
\text { metropolitan } \\
\text { area for } \\
\text { diversity }\end{array}$ & $\begin{array}{l}\text { Not } \\
\text { reported }\end{array}$ & $\begin{array}{l}\text { Not } \\
\text { reported }\end{array}$ \\
\hline $\begin{array}{l}\text { Simpso } \\
\text { n } 2002 \\
23\end{array}$ & $\begin{array}{l}\text { New } \\
\text { Jersey / } \\
\text { Pennsylv } \\
\text { ania, } \\
\text { USA }\end{array}$ & $\begin{array}{l}\text { Predomina } \\
\text { ntly white } \\
\text { (77\% and } \\
83 \% \text {, } \\
\text { respectivel } \\
\text { y in each } \\
\text { phase) }\end{array}$ & $\begin{array}{l}\text { Not } \\
\text { reporte } \\
\text { d }\end{array}$ & $\begin{array}{l}\text { Femal } \\
\mathrm{e} \\
(71 \% \\
\text { and } \\
69 \%, \\
\text { in } \\
\text { each } \\
\text { phase } \\
\text { ) }\end{array}$ & $\begin{array}{l}\text { Not } \\
\text { reporte } \\
\text { d }\end{array}$ & 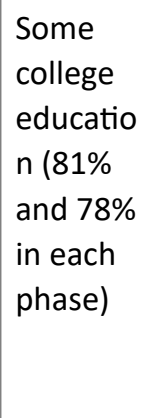 & Not reported & $\begin{array}{l}\text { Not } \\
\text { reported }\end{array}$ & $\begin{array}{l}\text { Ages } 35 \\
\text { to } 44 \\
\text { (phase I; } \\
52 \% \text { ) and } \\
25 \text { to } 34 \\
\text { years } \\
\text { (phase II; } \\
56 \% \text { ) }\end{array}$ \\
\hline $\begin{array}{l}\text { Lee } \\
200324\end{array}$ & $\begin{array}{l}\text { Seattle, } \\
\text { USA }\end{array}$ & $\begin{array}{l}\text { Spanish } \\
\text { Speaking }\end{array}$ & $\begin{array}{l}\text { Not } \\
\text { reporte } \\
\text { d }\end{array}$ & $\begin{array}{l}\text { All } \\
\text { but } \\
\text { two } \\
\text { were } \\
\text { femal } \\
\text { e }\end{array}$ & $\begin{array}{l}\text { Not } \\
\text { reporte } \\
\text { d }\end{array}$ & $\begin{array}{l}\text { Not } \\
\text { reporte } \\
\text { d }\end{array}$ & Not reported & $\begin{array}{l}\text { Not } \\
\text { reported }\end{array}$ & $\begin{array}{l}\text { Not } \\
\text { reported }\end{array}$ \\
\hline $\begin{array}{l}\text { Agran } \\
200425\end{array}$ & $\begin{array}{l}\text { Californi } \\
\text { a, USA }\end{array}$ & $\begin{array}{l}\text { Not } \\
\text { reported }\end{array}$ & $\begin{array}{l}\text { Not } \\
\text { reporte } \\
\text { d }\end{array}$ & $\begin{array}{l}\text { Not } \\
\text { report } \\
\text { ed }\end{array}$ & $\begin{array}{l}\text { Not } \\
\text { reporte } \\
\text { d }\end{array}$ & $\begin{array}{l}\text { Not } \\
\text { reporte } \\
\text { d }\end{array}$ & Not reported & $\begin{array}{l}\text { Not } \\
\text { reported }\end{array}$ & $\begin{array}{l}\text { Not } \\
\text { reported }\end{array}$ \\
\hline
\end{tabular}




\begin{tabular}{|c|c|c|c|c|c|c|c|c|c|}
\hline Study & $\begin{array}{l}\text { Place of } \\
\text { residenc } \\
\mathbf{e}\end{array}$ & $\begin{array}{l}\text { Race/ } \\
\text { ethnicity/ } \\
\text { culture/ } \\
\text { language }\end{array}$ & $\begin{array}{l}\text { Occupa } \\
\text { tion }\end{array}$ & $\begin{array}{l}\text { Gend } \\
\text { er/ } \\
\text { sex }\end{array}$ & $\begin{array}{l}\text { Religio } \\
\mathrm{n}\end{array}$ & $\begin{array}{l}\text { Educati } \\
\text { on }\end{array}$ & $\begin{array}{l}\text { Socio- } \\
\text { economic } \\
\text { Status }\end{array}$ & $\begin{array}{l}\text { Social } \\
\text { capital }\end{array}$ & Plus \\
\hline $\begin{array}{l}\text { Lenno } \\
\text { n } 2007 \\
26\end{array}$ & $\begin{array}{l}\text { Brisbane, } \\
\text { Australia }\end{array}$ & $\begin{array}{l}\text { Most were } \\
\text { Caucasian }\end{array}$ & $\begin{array}{l}\text { Not } \\
\text { reporte } \\
\text { d }\end{array}$ & $\begin{array}{l}21 \\
\text { moth } \\
\text { ers } \\
\text { and } 3 \\
\text { father } \\
\mathrm{s}\end{array}$ & $\begin{array}{l}\text { Not } \\
\text { reporte } \\
\text { d }\end{array}$ & $\begin{array}{l}\text { Most } \\
\text { parents } \\
\text { had at } \\
\text { least } \\
\text { some } \\
\text { post- } \\
\text { seconda } \\
\text { ry } \\
\text { educatio } \\
\mathrm{n} \\
(20 / 24)\end{array}$ & $\begin{array}{l}\text { Centres for } \\
\text { recruitment } \\
\text { were } \\
\text { selected } \\
\text { based on SES } \\
\text { indicators of } \\
\text { the } \\
\text { surrounding } \\
\text { suburbs (one } \\
\text { in an upper } \\
\text { SES area; one } \\
\text { drawing on } \\
\text { lower SES } \\
\text { suburbs). } \\
\text { Around half } \\
\text { (13/24) of } \\
\text { the parents } \\
\text { indicating } \\
\text { family } \\
\text { income. } \\
\text { \$A60 000 per } \\
\text { annum } \\
\text { (consistent } \\
\text { with national } \\
\text { figures for } \\
\text { median } \\
\text { income for } \\
\text { couple- } \\
\text { families with } \\
\text { children) }\end{array}$ & $\begin{array}{l}\text { Not } \\
\text { reported }\end{array}$ & $\begin{array}{l}\text { Most } \\
\text { parents } \\
\text { were } \\
\text { aged } \\
\text { between } \\
30 \text { and } \\
39 \text { years } \\
(16 / 24) ; \\
\text { two step } \\
\text { families } \\
\text { and at } \\
\text { least two } \\
\text { sole- } \\
\text { parent } \\
\text { families }\end{array}$ \\
\hline $\begin{array}{l}\text { Medoff } \\
- \\
\text { Cooper } \\
200728\end{array}$ & $\begin{array}{l}\text { Large } \\
\text { mid- } \\
\text { Atlantic } \\
\text { city, USA }\end{array}$ & $\begin{array}{l}\text { One focus } \\
\text { group } \\
\text { consisted } \\
\text { of } 8 \text { White } \\
\text { women; } \\
\text { the other } \\
\text { focus group } \\
\text { consisted } \\
\text { of } 4 \text { African } \\
\text { American } \\
\text { women } \\
\text { and } 4 \\
\text { Latina } \\
\text { women. }\end{array}$ & $\begin{array}{l}\text { Most } \\
\text { particip } \\
\text { ants } \\
\text { were } \\
\text { employe } \\
d \text { either } \\
\text { full time } \\
\text { or part } \\
\text { time }\end{array}$ & $\begin{array}{l}\text { All } \\
\text { femal } \\
\text { e }\end{array}$ & $\begin{array}{l}\text { Not } \\
\text { reporte } \\
\text { d }\end{array}$ & $\begin{array}{l}\text { At least } \\
\text { a high } \\
\text { school } \\
\text { educatio } \\
\mathrm{n}\end{array}$ & $\begin{array}{l}\text { Household } \\
\text { income of at } \\
\text { least } \$ 20,000\end{array}$ & $\begin{array}{l}\text { Not } \\
\text { reported }\end{array}$ & $\begin{array}{l}\text { Not } \\
\text { reported }\end{array}$ \\
\hline
\end{tabular}




\begin{tabular}{|c|c|c|c|c|c|c|c|c|c|}
\hline Study & $\begin{array}{l}\text { Place of } \\
\text { residenc } \\
\mathrm{e}\end{array}$ & $\begin{array}{l}\text { Race/ } \\
\text { ethnicity/ } \\
\text { culture/ } \\
\text { language }\end{array}$ & $\begin{array}{l}\text { Occupa } \\
\text { tion }\end{array}$ & $\begin{array}{l}\text { Gend } \\
\text { er/ } \\
\text { sex }\end{array}$ & $\begin{array}{l}\text { Religio } \\
n\end{array}$ & $\begin{array}{l}\text { Educati } \\
\text { on }\end{array}$ & $\begin{array}{l}\text { Socio- } \\
\text { economic } \\
\text { Status }\end{array}$ & $\begin{array}{l}\text { Social } \\
\text { capital }\end{array}$ & Plus \\
\hline $\begin{array}{l}\text { Winsto } \\
\text { n } 2007 \\
29\end{array}$ & $\begin{array}{l}\text { Philadelp } \\
\text { hia, USA }\end{array}$ & $\begin{array}{l}\text { Formative } \\
\text { phase: } \\
\text { African } \\
\text { American } \\
46.7 \% \text {, } \\
\text { white } \\
17.8 \% \text {, } \\
\text { Hispanic/ } \\
\text { Latino } \\
35.5 \% \text {. } \\
\text { Evaluative } \\
\text { phase: } \\
\text { African } \\
\text { American } \\
56.3 \%, \\
\text { white } \\
18.8 \% \text {, } \\
\text { Hispanic/ } \\
\text { Latino25.0 } \\
\%\end{array}$ & $\begin{array}{l}\text { Among } \\
\text { those } \\
\text { employe } \\
d \\
\text { formativ } \\
\text { e phase } \\
\text { - 44.9\% } \\
\text { were in } \\
\text { service } \\
\text { and } \\
20.4 \% \\
\text { administ } \\
\text { rative } \\
\text { evaluati } \\
\text { ve } \\
\text { phase - } \\
33 \% \text { was } \\
\text { in } \\
\text { service } \\
\text { and } 44 \% \\
\text { administ } \\
\text { rative }\end{array}$ & $\begin{array}{l}86.9 \% \\
\text { femal } \\
\text { e in } \\
\text { forma } \\
\text { tive } \\
\text { phase } \\
; \\
89.8 \% \\
\text { in } \\
\text { evalu } \\
\text { ative }\end{array}$ & $\begin{array}{l}\text { Not } \\
\text { reporte } \\
\text { d }\end{array}$ & $\begin{array}{l}\text { Inclusio } \\
\mathrm{n} \\
\text { criteria } \\
\text { was } \\
\text { educatio } \\
\text { nal } \\
\text { attainm } \\
\text { ent of, } \\
\text { at most, } \\
\text { at most } \\
\text { a high } \\
\text { school } \\
\text { diploma }\end{array}$ & $\begin{array}{l}\text { Unemployed } \\
50.5 \% \text { in } \\
\text { formative } \\
\text { phase; } 43.2 \% \\
\text { in evaluative } \\
\text { phase }\end{array}$ & $\begin{array}{l}\text { Not } \\
\text { reported }\end{array}$ & $\begin{array}{l}\text { Formativ } \\
\text { e phase: } \\
30.8 \% \\
\text { single, } \\
5.6 \% \\
\text { separate } \\
\text { d, } 3.7 \% \\
\text { divorced } \\
\text { Evaluativ } \\
\text { e phase: } \\
45.5 \% \\
\text { single, } 4 \\
\% \\
\text { separate } \\
\text { d, } 5.3 \% \\
\text { divorced }\end{array}$ \\
\hline $\begin{array}{l}\text { Johnst } \\
\text { on } \\
200930\end{array}$ & $\begin{array}{l}\text { Central } \\
\text { and } \\
\text { southeas } \\
\text { t Seattle, } \\
\text { USA }\end{array}$ & $\begin{array}{l}\text { Only } \\
\text { African } \\
\text { American, } \\
\text { Somali, and } \\
\text { Vietnames } \\
\text { e parents } \\
\text { were } \\
\text { recruited. }\end{array}$ & $\begin{array}{l}\text { Not } \\
\text { reporte } \\
\text { d }\end{array}$ & $\begin{array}{l}80 \% \\
\text { femal } \\
\text { e } \\
\text { overal } \\
\text { I } \\
\text { (disag } \\
\text { gregat } \\
\text { ed by } \\
\text { race } \\
\text { report } \\
\text { ed) }\end{array}$ & $\begin{array}{l}\text { Not } \\
\text { reporte } \\
\text { d }\end{array}$ & $\begin{array}{l}\text { Not } \\
\text { reporte } \\
\text { d }\end{array}$ & $\begin{array}{l}\text { Low income } \\
\text { groups were } \\
\text { specifically } \\
\text { recruited for } \\
\text { the study; } \\
63 \% \text { own a } \\
\text { vehicle; } \\
\text { (disaggregate } \\
\text { d by race } \\
\text { reported) }\end{array}$ & $\begin{array}{l}\text { Not } \\
\text { reported }\end{array}$ & $\begin{array}{l}\text { Are likely } \\
\text { to be } \\
\text { immigra } \\
\text { nts }\end{array}$ \\
\hline
\end{tabular}




\begin{tabular}{|c|c|c|c|c|c|c|c|c|c|}
\hline Study & $\begin{array}{l}\text { Place of } \\
\text { residenc } \\
\mathrm{e}\end{array}$ & $\begin{array}{l}\text { Race/ } \\
\text { ethnicity/ } \\
\text { culture/ } \\
\text { language }\end{array}$ & $\begin{array}{l}\text { Occupa } \\
\text { tion }\end{array}$ & $\begin{array}{l}\text { Gend } \\
\text { er/ } \\
\text { sex }\end{array}$ & $\begin{array}{l}\text { Religio } \\
\mathrm{n}\end{array}$ & $\begin{array}{l}\text { Educati } \\
\text { on }\end{array}$ & $\begin{array}{l}\text { Socio- } \\
\text { economic } \\
\text { Status }\end{array}$ & $\begin{array}{l}\text { Social } \\
\text { capital }\end{array}$ & Plus \\
\hline $\begin{array}{l}\text { Erkobo } \\
\text { ni } 2010 \\
31\end{array}$ & $\begin{array}{l}\text { Beijing } \\
\text { China }\end{array}$ & $\begin{array}{l}\text { Not } \\
\text { reported }\end{array}$ & $\begin{array}{l}8.5 \% \\
\text { were } \\
\text { unempl } \\
\text { oyed } \\
\text { and rest } \\
\text { employe } \\
d(12.5 \% \\
\text { medical, } \\
12.5 \% \\
\text { administ } \\
\text { rative, } \\
37.5 \% \\
\text { service) }\end{array}$ & $\begin{array}{l}\text { Not } \\
\text { report } \\
\text { ed }\end{array}$ & $\begin{array}{l}\text { Not } \\
\text { reporte } \\
\text { d }\end{array}$ & $\begin{array}{l}\text { Not } \\
\text { reporte } \\
\text { d }\end{array}$ & Not reported & $\begin{array}{l}\text { Not } \\
\text { reported }\end{array}$ & $\begin{array}{l}\text { Aged } 31 \\
\text { to } 35 \\
(56.5 \%) \\
\text { and } \\
\text { married } \\
(98.6 \%)\end{array}$ \\
\hline
\end{tabular}




\begin{tabular}{|c|c|c|c|c|c|c|c|c|c|}
\hline Study & $\begin{array}{l}\text { Place of } \\
\text { residenc } \\
\mathrm{e}\end{array}$ & $\begin{array}{l}\text { Race/ } \\
\text { ethnicity/ } \\
\text { culture/ } \\
\text { language }\end{array}$ & $\begin{array}{l}\text { Occupa } \\
\text { tion }\end{array}$ & $\begin{array}{l}\text { Gend } \\
\text { er/ } \\
\text { sex }\end{array}$ & $\begin{array}{l}\text { Religio } \\
\mathrm{n}\end{array}$ & $\begin{array}{l}\text { Educati } \\
\text { on }\end{array}$ & $\begin{array}{l}\text { Socio- } \\
\text { economic } \\
\text { Status }\end{array}$ & $\begin{array}{l}\text { Social } \\
\text { capital }\end{array}$ & Plus \\
\hline $\begin{array}{l}\text { Brown } \\
201332\end{array}$ & $\begin{array}{l}\text { New } \\
\text { South } \\
\text { Wales, } \\
\text { Australia }\end{array}$ & $\begin{array}{l}11 \\
\text { language } \\
\text { specific } \\
\text { focus } \\
\text { groups in } \\
\text { Arabic, } \\
\text { Assyrian, } \\
\text { Cantonese, } \\
\text { Mandarin, } \\
\text { Vietnames } \\
\text { e and } \\
\text { Turkish. }\end{array}$ & $\begin{array}{l}\text { Not } \\
\text { reporte } \\
\text { d }\end{array}$ & $\begin{array}{l}\text { Ten } \\
\text { group } \\
\mathrm{s} \\
\text { were } \\
\text { femal } \\
\text { e-only } \\
\text { and } \\
\text { one } \\
\text { Arabic } \\
- \\
\text { speaki } \\
\text { ng } \\
\text { group } \\
\text { was } \\
\text { male- } \\
\text { only }\end{array}$ & $\begin{array}{l}\text { Not } \\
\text { reporte } \\
\text { d }\end{array}$ & $\begin{array}{l}\text { Not } \\
\text { reporte } \\
\text { d }\end{array}$ & Not reported & $\begin{array}{l}\text { Not } \\
\text { reported }\end{array}$ & $\begin{array}{l}\text { All } \\
\text { participa } \\
\text { nts were } \\
\text { non- } \\
\text { English } \\
\text { speaking } \\
\text { in } \\
\text { Australia } \\
\text { and the } \\
\text { study } \\
\text { was } \\
\text { hence } \\
\text { focussed } \\
\text { on a } \\
\text { linguistic } \\
\text { minority. } \\
\text { However, } \\
\text { majority } \\
\text { also } \\
\text { spoke } \\
\text { English } \\
\text { in Arabic } \\
\text { and } \\
\text { Assyrain } \\
\text { group } \\
\text { only. } \\
\text { Many of } \\
\text { them are } \\
\text { immigra } \\
\text { nts } \\
\text { (mean } \\
\text { duration } \\
\text { in } \\
\text { Australia } \\
\text { is } 10-20 \\
\text { years in } \\
\text { different } \\
\text { groups } \\
\text { with the } \\
\text { minimu } \\
\text { m being } \\
\text { one year } \\
\text { in } \\
\text { anyrian }\end{array}$ \\
\hline
\end{tabular}




\begin{tabular}{|c|c|c|c|c|c|c|c|c|c|}
\hline Study & $\begin{array}{l}\text { Place of } \\
\text { residenc } \\
\mathrm{e}\end{array}$ & $\begin{array}{l}\text { Race/ } \\
\text { ethnicity/ } \\
\text { culture/ } \\
\text { language }\end{array}$ & $\begin{array}{l}\text { Occupa } \\
\text { tion }\end{array}$ & $\begin{array}{l}\text { Gend } \\
\text { er/ } \\
\text { sex }\end{array}$ & $\begin{array}{l}\text { Religio } \\
\mathrm{n}\end{array}$ & $\begin{array}{l}\text { Educati } \\
\text { on }\end{array}$ & $\begin{array}{l}\text { Socio- } \\
\text { economic } \\
\text { Status }\end{array}$ & $\begin{array}{l}\text { Social } \\
\text { capital }\end{array}$ & Plus \\
\hline $\begin{array}{l}\text { Chen } \\
201434\end{array}$ & $\begin{array}{l}\text { Shanghai } \\
\text {, China }\end{array}$ & $\begin{array}{l}\text { Not } \\
\text { reported }\end{array}$ & $\begin{array}{l}\text { Not } \\
\text { reporte } \\
\text { d }\end{array}$ & $\begin{array}{l}\text { Two } \\
\text { of the } \\
14 \\
\text { partici } \\
\text { pants } \\
\text { were } \\
\text { father } \\
S \text { and } \\
\text { the } \\
\text { rest } \\
\text { were } \\
\text { moth } \\
\text { ers }\end{array}$ & $\begin{array}{l}\text { Not } \\
\text { reporte } \\
\text { d }\end{array}$ & $\begin{array}{l}\text { Bachelor } \\
\text { s or } \\
\text { higher }\end{array}$ & $\begin{array}{l}\text { Monthly } \\
\text { income at or } \\
\text { above } \\
\text { average of } \\
\text { city ; all } \\
\text { drove to } \\
\text { work using } \\
\text { car }\end{array}$ & $\begin{array}{l}\text { Not } \\
\text { reported }\end{array}$ & $\begin{array}{l}\text { Young } \\
\text { age } 29 \text { to } \\
34 \text { years }\end{array}$ \\
\hline $\begin{array}{l}\text { Nelson } \\
201535\end{array}$ & $\begin{array}{l}\text { Riyadh, } \\
\text { Saudi } \\
\text { Arabia }\end{array}$ & $\begin{array}{l}\text { All spoke } \\
\text { Arabic }\end{array}$ & $\begin{array}{l}\text { Not } \\
\text { reporte } \\
\text { d }\end{array}$ & $\begin{array}{l}\text { All } \\
\text { femal } \\
\text { e }\end{array}$ & $\begin{array}{l}\text { Not } \\
\text { reporte } \\
\text { d } \\
\text { specific } \\
\text { ally but } \\
\text { mentio } \\
\text { ns } \\
\text { predo } \\
\text { minant } \\
\text { ly } \\
\text { Muslim } \\
\text { popula } \\
\text { tion as } \\
\text { influen } \\
\text { cing } \\
\text { percep } \\
\text { tion. }\end{array}$ & $\begin{array}{l}\text { Not } \\
\text { reporte } \\
\text { d }\end{array}$ & Not reported & $\begin{array}{l}\text { Not } \\
\text { reported }\end{array}$ & $\begin{array}{l}\text { Only } \\
\text { pregnant } \\
\text { women. } \\
\text { Over } \\
50 \% \text { of } \\
\text { the } \\
\text { participa } \\
\text { nts were } \\
\text { in the } \\
\text { second } \\
\text { half of } \\
\text { pregnanc } \\
\text { y. For } 7 \\
\text { women } \\
\text { this was } \\
\text { their first } \\
\text { pregnanc } \\
\text { y, and } \\
\text { the rest } \\
\text { already } \\
\text { had } \\
\text { children }\end{array}$ \\
\hline
\end{tabular}




\begin{tabular}{|c|c|c|c|c|c|c|c|c|c|}
\hline Study & $\begin{array}{l}\text { Place of } \\
\text { residenc } \\
\mathrm{e}\end{array}$ & $\begin{array}{l}\text { Race/ } \\
\text { ethnicity/ } \\
\text { culture/ } \\
\text { language }\end{array}$ & $\begin{array}{l}\text { Occupa } \\
\text { tion }\end{array}$ & $\begin{array}{l}\text { Gend } \\
\text { er/ } \\
\text { sex }\end{array}$ & $\begin{array}{l}\text { Religio } \\
\mathrm{n}\end{array}$ & $\begin{array}{l}\text { Educati } \\
\text { on }\end{array}$ & $\begin{array}{l}\text { Socio- } \\
\text { economic } \\
\text { Status }\end{array}$ & $\begin{array}{l}\text { Social } \\
\text { capital }\end{array}$ & Plus \\
\hline $\begin{array}{l}\text { Liu } \\
201637\end{array}$ & $\begin{array}{l}\text { Shantou, } \\
\text { China }\end{array}$ & $\begin{array}{l}\text { Not } \\
\text { reported }\end{array}$ & $\begin{array}{l}\text { Not } \\
\text { reporte } \\
\text { d }\end{array}$ & $\begin{array}{l}\text { Not } \\
\text { report } \\
\text { ed }\end{array}$ & $\begin{array}{l}\text { Not } \\
\text { reporte } \\
\text { d }\end{array}$ & $\begin{array}{l}\text { Not } \\
\text { reporte } \\
\mathrm{d} \text { but all } \\
\text { particip } \\
\text { ants } \\
\text { owned } \\
\text { at least } \\
\text { one } \\
\text { private } \\
\text { car }\end{array}$ & $\begin{array}{l}\text { Twenty-five } \\
\text { of the } 30 \\
\text { participants } \\
\text { were native } \\
\text { to Shantou, } \\
\text { and the rest } \\
\text { were } \\
\text { originally } \\
\text { from other } \\
\text { parts of } \\
\text { China }\end{array}$ & $\begin{array}{l}\text { Not } \\
\text { reported }\end{array}$ & $\begin{array}{l}\text { Only } \\
\text { pregnant } \\
\text { women } \\
\text { were } \\
\text { recruited } \\
.76 .7 \% \\
\text { were } \\
\text { pregnant } \\
\text { for the } \\
\text { first time }\end{array}$ \\
\hline $\begin{array}{l}\text { Fleishe } \\
\text { r } 2017 \\
38\end{array}$ & USA & $\begin{array}{l}\text { African } \\
\text { American } \\
(48 \%)\end{array}$ & $\begin{array}{l}\text { Not } \\
\text { reporte } \\
\text { d }\end{array}$ & $\begin{array}{l}\text { Femal } \\
\text { e } \\
(86 \%)\end{array}$ & $\begin{array}{l}\text { Not } \\
\text { reporte } \\
\text { d }\end{array}$ & $\begin{array}{l}\text { At least } \\
\text { some } \\
\text { college } \\
\text { educatio } \\
\mathrm{n}(62 \%)\end{array}$ & $\begin{array}{l}\text { Primary care } \\
\text { practices } \\
\text { were chosen } \\
\text { based on } \\
\text { geographic } \\
\text { location to } \\
\text { ensure } \\
\text { variability in } \\
\text { participant } \\
\text { race/ } \\
\text { ethnicity, } \\
\text { community } \\
\text { setting and } \\
\text { socioeconom } \\
\text { ic status }\end{array}$ & $\begin{array}{l}\text { Not } \\
\text { reported }\end{array}$ & $\begin{array}{l}\text { Not } \\
\text { reported }\end{array}$ \\
\hline $\begin{array}{l}\text { Hunter } \\
201739\end{array}$ & $\begin{array}{l}\text { New } \\
\text { South } \\
\text { Wales, } \\
\text { Australia }\end{array}$ & $\begin{array}{l}\text { Aboriginal } \\
\text { but spoke } \\
\text { English as } \\
\text { their } \\
\text { primary } \\
\text { language at } \\
\text { home }\end{array}$ & $\begin{array}{l}\text { Not } \\
\text { reporte } \\
\text { d }\end{array}$ & $\begin{array}{l}\text { All } \\
\text { were } \\
\text { femal } \\
\text { e }\end{array}$ & $\begin{array}{l}\text { From } \\
\text { differe } \\
\text { nt } \\
\text { educati } \\
\text { onal } \\
\text { strata; } \\
\text { almost } \\
\text { equally } \\
\text {. }\end{array}$ & $\begin{array}{l}\text { Not } \\
\text { reporte } \\
\text { d }\end{array}$ & Not reported & $\begin{array}{l}\text { Not } \\
\text { reported }\end{array}$ & $\begin{array}{l}\text { Not } \\
\text { reported }\end{array}$ \\
\hline
\end{tabular}




\begin{tabular}{|c|c|c|c|c|c|c|c|c|c|}
\hline Study & $\begin{array}{l}\text { Place of } \\
\text { residenc } \\
\mathrm{e}\end{array}$ & $\begin{array}{l}\text { Race/ } \\
\text { ethnicity/ } \\
\text { culture/ } \\
\text { language }\end{array}$ & $\begin{array}{l}\text { Occupa } \\
\text { tion }\end{array}$ & $\begin{array}{l}\text { Gend } \\
\text { er/ } \\
\text { sex }\end{array}$ & $\begin{array}{l}\text { Religio } \\
\mathrm{n}\end{array}$ & $\begin{array}{l}\text { Educati } \\
\text { on }\end{array}$ & $\begin{array}{l}\text { Socio- } \\
\text { economic } \\
\text { Status }\end{array}$ & $\begin{array}{l}\text { Social } \\
\text { capital }\end{array}$ & Plus \\
\hline $\begin{array}{l}\text { McKen } \\
\text { zie } \\
201740\end{array}$ & $\begin{array}{l}\text { Columbi } \\
\text { a, USA }\end{array}$ & $\begin{array}{l}\text { Somali } \\
\text { refugees } \\
\text { and } \\
\text { immigrants } \\
\text { in USA }\end{array}$ & $\begin{array}{l}\text { Key } \\
\text { informa } \\
\text { nt } \\
\text { intervie } \\
\text { ws } \\
\text { revealed } \\
\text { un- } \\
\text { employ } \\
\text { ment is } \\
\text { a } \\
\text { problem } \\
\text { in the } \\
\text { commu } \\
\text { nity }\end{array}$ & $\begin{array}{l}\text { Not } \\
\text { report } \\
\text { ed }\end{array}$ & $\begin{array}{l}\text { Not } \\
\text { reporte } \\
\text { d }\end{array}$ & $\begin{array}{l}\text { Key } \\
\text { informa } \\
\text { nt } \\
\text { review } \\
\text { revealed } \\
\text { that the } \\
\text { commu } \\
\text { nity has } \\
\text { a } \\
\text { problem } \\
\text { of } \\
\text { illiteracy }\end{array}$ & $\begin{array}{l}\text { Not reported } \\
\text { but says the } \\
\text { community } \\
\text { has a } \\
\text { problem of } \\
\text { daily living }\end{array}$ & $\begin{array}{l}\text { Key } \\
\text { informant } \\
\text { reviews } \\
\text { mentions } \\
\text { says the } \\
\text { communit } \\
\text { y has a } \\
\text { problem of } \\
\text { adjustmen } \\
\text { t and } \\
\text { culture } \\
\text { shock }\end{array}$ & $\begin{array}{l}\text { Key } \\
\text { informan } \\
\mathrm{t} \\
\text { interview } \\
\mathrm{s} \\
\text { revealed } \\
\text { that time } \\
\text { send in } \\
\text { refugee } \\
\text { camps } \\
\text { and } \\
\text { displace } \\
\mathrm{d} \text { status } \\
\text { has led } \\
\text { to } \\
\text { mental } \\
\text { health } \\
\text { issues }\end{array}$ \\
\hline $\begin{array}{l}\text { Hall } \\
201841\end{array}$ & $\begin{array}{l}\text { Sydney, } \\
\text { Australia }\end{array}$ & $\begin{array}{l}\text { Culturally } \\
\text { and } \\
\text { linguisticall } \\
\text { y diverse } \\
\text { group } \\
\text { included } \\
\text { (also see } \\
\text { education } \\
\text { and SES } \\
\text { column) }\end{array}$ & $\begin{array}{l}\text { Not } \\
\text { Reporte } \\
\text { d }\end{array}$ & $\begin{array}{l}95 \% \\
\text { were } \\
\text { femal } \\
\text { e }\end{array}$ & $\begin{array}{l}\text { Not } \\
\text { Report } \\
\text { ed }\end{array}$ & \multicolumn{2}{|c|}{$\begin{array}{l}\text { Three groups of child } \\
\text { restraint system (CRS) } \\
\text { users: } \\
\text { (a) Participants in high } \\
\text { income and high } \\
\text { education brackets } \\
\text { (high SES) (b) } \\
\text { Participants in lower } \\
\text { income and lower } \\
\text { education brackets (low } \\
\text { SES) and } \\
\text { (c) Participants from a } \\
\text { culturally and } \\
\text { linguistically diverse } \\
\text { background (CALD }\end{array}$} & $\begin{array}{l}\text { Not } \\
\text { Reported }\end{array}$ & $\begin{array}{l}\text { Not } \\
\text { Reported }\end{array}$ \\
\hline
\end{tabular}




\section{Syntheses of findings}

Five key themes emerged from the thematic analysis of the included studies: (1) Perceived risk for injuries and perceived safety benefit of child restraint usage varies in different settings and between different types of caregivers. (2) Practical issues around the use of child restraints was a major barrier to its uptake as a child safety measure. (3) Restraint use was considered a mechanism to discipline children rather than a safety device by parents and as children became older, they actively seek opportunities to negotiate non-usage of restraint. (4) Adoption and enforcement of laws shaped perceptions and usage in all settings. (5) Perceptions and norms of child safety differed amongst culturally and linguistically diverse groups.

\section{Perceived risk for injuries and perceived safety benefit of child restraint usage varies in different setting and between different types of caregivers}

A mixture of perceived risk of injuries and the perceived safety benefit of using child restraints to prevent road traffic injuries influenced uptake of restraints and this varied not only across different contexts but also between different types of care givers.

Many studies $21232628-3032343537$ included participants who acknowledged that it was "dangerous for children to travel unrestrained" 32 owing to a high risk of injuries. Participants also reported that the risk of injuries was dependent on the duration of rides, speed, driver experience (being 'safe drivers') and 'safe areas' 21232528303439 . Authors reported that among non-users of child restraints, child restraint use was determined by risk perception with authors concluding that a "short distance and slow speed in the city would not cause serious injury even in a crash; CSS is not that important." This was evidenced by the quote from parents "We don't ask my child to wear the seat belt because most of the time we are driving in the downtown area" 34 . Mothers often mentioned that fathers, grandparents and other caregivers had lower risk perceptions and allowed children to be transported unrestrained which made enforcement difficult. In one study 24 , "mothers felt it was important for more educational efforts targeted specifically at fathers, who often were working and therefore not present during potential teachable moments such as doctor visits, or school and community events". Often fathers played an important role in decision-making on buying restraints, and convincing them was often difficult due to a lack of knowledge "I heard it and want to buy it, but I fail to persuade my husband because I don't know much about it" (non-restraint user mother) ${ }^{34}$. Participants in the study from Saudi Arabia mentioned that, "Allah is in control of everything that happens, and it is all in His hands. Whether or not I use a car seat will not matter"35. Similar thoughts were expressed by Arab-speaking male participants in another study 32 .

The perceived safety benefits of child restraints was low in many settings 30-32 34. Concerns were raised about the balance between "being belted versus the risks of being trapped in the car" 3037 . Participants often expressed doubts on the preventability of particular types of restraints or belt-positioning. Booster seats and forward-facing restraints were mentioned to be untrustworthy by participants; "I don't think they [belt-positioning booster seats] are safe-they are more dangerous than a car seat [forward-facing restraint]" 26. Cultural notions on what constitutes safety was also reflected when participants mentioned that holding children on laps provided better safety in case of a crash - "It is a natural human response that people would hold children tightly when a car brakes. If a child is restrained in a safety seat, I feel I could not protect my child when a car suddenly brakes" 37. 


\section{Practical issues around the use of child restraints was a major barrier to its uptake as a child safety measure}

Participants in all studies mentioned several practical barriers in using child restraints. Cost was almost a universal cause of concern which limited use 2123242629303239 . Educational sessions were thought to be useful when accompanied by discounts or giveaways of restraints. Rebates were thought to be an impractical method because of the difficulty in obtaining rebates and the time delay. Discounts were also reported to be inadequate ${ }^{21}$. A few studies reported the need for them to be provided completely free 3840 . Interestingly, costs were not reported to be a barrier in three studies from China. The difference in studies from China might be due to the fact that most participants in these studies had jobs or were earning at or above the average wage in the city (one of these studies did not report on socio-economic or job status but authors reported cost was not a barrier) 313437.

The issue of cost was also related to other practical constraints like family size. Restraints were thought to be like, "sardines in the back seat.... [The car seats] take up too much space. In particular, full-back booster seats allow for only two children in a mid-sized car, with no room for extra passengers" 28 . This was particularly problematic in cultures which do not have nuclear family set-ups or had large family sizes and in communities where parenting responsibilities were often shared or where providing or taking lifts was common 2425 28-30 3235 . One study from Australia mentioned the importance of "learning from the authorised fitter while others felt paying for an authorised restraint fitter to fit the car seat was a waste of money" 39 .

The practicalities around restraints as children grow up and the need for frequent transitioning after a particular age or when they have attained a particular weight or height was identified as a major practical issue for use by participants in seven studies 21232628323839 . Some of these studies highlighted the lack of knowledge about transition as well as different viewpoints among parents about the suitability of such legislation for older children.

The comfort of children was also raised as an important consideration in many studies 28323435 . Comfort often outweighed safety concerns leading to non-usage: "when the kid's on the restraint actually crying a lot, crying like crazy, and at that moment if you cannot stop him crying, especially on a long journey" 32. The only study that included children as participants reported that most of their comments were about comfort and that they "liked ones that were large enough for them and disliked ones that seemed or felt 'too skinny'. Comfort seems to be a significant issue for children"23. As such, comfort of children seems to be an important practical enabler to increased use of restraints. Few studies had parents mentioning that booster seats provided a better view of the outside to their children, thus improving compliance 21 28. Other issues that limited use of child restraints were lack of availability, incompatibility between cars and booster seat designs, lack of shoulder belts in the rear seats of older vehicles, and the time consuming and complicated nature of buckling children 212324262832 .

\section{Restraint use is considered a mechanism to discipline children rather than a safety device by parents and as children grow older, they actively seek opportunities to negotiate non-usage of restraints}

Many parents saw the use of restraints as more of an issue of disciplining their children. This meant that parents often relaxed rules for restraints as need be. For example, several parents had commented that they had an "obedient child, and therefore the parent could let the child use a lap belt" 21 . They also "gave in to children who resisted CSS use, often citing a need to keep the child quiet to avoid distracting the driver" 25 . Studies also reported that parents occasionally "would allow a child to travel in the front seat as a special treat or because the trip was perceived as a short one... (with a) desire to support children in their maturation and perceiving the move to the front seat as part of this: 'there's a sense 
that your kid is growing up and you kind of want to-it feels good when they're taking their little steps like that and I guess subconsciously you weaken'"26.

As children grew older (reported around 3-5 years in different studies), peer pressure and the desire for children to be perceived as a "big kid" was a major factor preventing restraint usage. This was particularly problematic because, "few older siblings or friends used booster seats" 21 and as such parents often found it "harder to resist as children grow older" 26 due to social norms and pressure"many said that after a certain age ( 4 or 5 years), children say that they feel like "babies" if they sit in a car seat" 28 .

How parents negotiated with children making these demands seemed to play an important role in child restraint use. Some parents could draw a distinction between safety (non-negotiable) and bath time and eating habits (negotiable), whereas others failed to do so and allowed children to negotiate on the use of restraints. Older children mostly learnt to unbuckle themselves too, which probably limited negotiability.

\section{Adoption and enforcement of laws shaped perceptions and usage in all settings}

The adoption and enforcement of laws mandating usage of restraints in children was identified as a very important factor influencing usage as well as influencing perceptions almost universally. This was true even when there was low awareness about the actual specifics of the law or there were gaps in the actual law (particularly around transition ages). Having a clear set of laws and guidelines which had been properly communicated leading to a high level of awareness was thought to be very important.

Enforcement of the law and perception of likelihood of enforcement were identified as important factors for compliance. For example, sometimes respondents "did not use the child seat restraint at night, thinking that a police officer could not see into the car in the dark" 25 .

Presence of the law and its enforcement not only shaped parents' perceptions but was also often used by parents to explain to children or negotiate with them. Children were often told that "Mommy will get in trouble" 28 and thus it was "easier for the kids to obey the police" 32. In a study from the USA, the African-American community was dismayed that the laws did not take equity into perspective - "It's always about money. If it is mandatory, it should be affordable" 30 .

\section{Perceptions and norms of child safety differed among culturally and linguistically diverse groups}

The use of an equity lens allowed us to explore some issues around equity but due to limitations in the availability of primary data, this could not be fully evaluated around all PROGRESS Plus domains. While all studies were from HIC or UMIC context, many studies focussed specifically on immigrants or culturally and linguistically diverse groups; one study ${ }^{39}$ was based in a regional area of a high income country with parents of low socio-economic status where approximately a third of participants were parents of Aboriginal children. Child safety in general was perceived very differently across groups implying that 'one-size fits all type of approach' might not be feasible when implementing programmes. As for example, immigrants mentioned "seatbelts and child safety restraints were not commonly used in their native countries, which may have contributed to the lack of awareness and low perception of risk among Latinos" 24 . Immigrants perceived booster seats to be a reflection of an "overprotective culture" or not being in alignment with their own culture in which "we love our kids. So we want to hug them.... hold them in our laps in cars" 32 . In fact, in the study specifically on Somali refugees and immigrants in USA, 
"child passenger safety, was never mentioned without prompting from the interviewer. Due to the abundance of other problems, safety issues are not top priority in the Somali community" 40 . The study from Saudi Arabia had mothers who felt, "this may be American culture, but in our culture, they will laugh at us if we place the child" (in a restraint) ${ }^{35}$. A study from USA 28 which explored differences in norms between White mothers and African-American and Latino mothers found, in addition to differences in usage, that the African-American and Latino mothers mentioned that the comfort of children when sleeping in a booster seat was a key reason for non-usage. African-American and Latina mothers also felt that their children did not like to sit in a child safety seat and this was an issue compared to white mothers who said this was not so much of a problem.

\section{Discussion}

Our narrative synthesis aimed at identifying the factors reported to influence the use of child restraints. The review was prospectively registered. We searched multiple databases and did not purposively sample within the studies we found.

We sought to understand the factors influencing the use of restraints from a wide section of stakeholders, including children but most of our studies focussed on parental perceptions only. Only one study involved a few children in a single phase of the study and none of the studies included policy makers, traffic policemen or others involved in formulating or implementing laws or policies around child restraints. Even among parents, mostly the mothers were involved and not fathers or other male family members, who often play important roles. In certain cultural and linguistic groups men are more often the drivers of motor vehicles. For example, in Saudi Arabia women have only been legally allowed to drive from 2018. As such, even sub-group analyses (such as type of participant, country income level, and type of qualitative study, i.e. associated with an intervention or stand-alone study) that we had planned a priori could not be conducted due to the paucity of data. We recommend future qualitative studies to include children and other participants to understand their perspectives. This would be particularly important for older children who actively negotiate for restraint exceptions with their parents.

The use of a PROGRESS Plus framework 17 as an equity lens in the current study is novel. PROGRESS-Plus has been used in quantitative systematic reviews and meta-analysis previously, to understand how an intervention is affected by equity factors. However, we are not aware of its use in qualitative systematic reviews. Although data on equity issues in the primary qualitative studies were scarce thereby limiting interpretation we contend that it is a feasible and essential element in qualitative evidence synthesis in order to explore how equity affects different social phenomenon and its interpretations. More methodological work on this is essential. This post-hoc approach was not planned during protocol phase but enabled us to understand the role of these equity parameters around this topic and demonstrate gaps in research in terms of equity. While differences based on gender and cultural and linguistically diverse backgrounds were evident, applying an equity lens through PROGRESS-plus broadens the scope to investigate factors that may influence child restraint use. This includes consideration of occupation, education, socio-economic status, social capital and personal circumstances (such as disability), relationship status (for example single/separated parents). Future qualitative studies should explore such factors to better understand child restraint usage. Most of the evidence we found was from high income countries and from big cities and this should be considered when making 
judgements on generalisability of the results of the studies to low- and middle- income countries or semi-urban areas, where different issues might affect the use of child restraints.

As in primary qualitative research, we reflected on how our backgrounds and positions might have influenced the conduct of this study. As a team, we decided on the choice of review topic through extensive deliberation over e-mail. We had initially planned to do a quantitative systematic review and meta-analyses but the availability of such studies in literature and feedback on the need for understanding the facilitators and barriers from team members in South Africa and India led to a refocussing of the review to target factors influencing restraint use. In our review, only a single author (SB) conducted all the data extraction and we acknowledge this as a limitation, although we verified extraction to ensure accuracy. As a team we had researchers and clinicians from the United Kingdom, South Africa, Australia and India, with expertise and experience in injury epidemiology, child injury and qualitative research. While it was not possible to involve the entire team in the analyses, formulation of themes was initially done by SB, refined with $\mathrm{KH}$ and then the final derivation of themes, interpretation of the results and critical feedback was done collectively by the entire team. We conducted a thematic synthesis of the included studies to investigate factors that impact child restraint use, which was in keeping with the epistemology of those studies as per the RETREAT criteria ${ }^{20}$. We have used standard methods to assess the quality of the included studies and saturation of themes was achieved. The quality of included qualitative studies varied and many did not report adequately on analysis methods or discussed issues around data saturation, positionality and reflexivity and some did not present quotes. We recommend future qualitative studies in this domain to use standardised reporting checklists like COREQ or SRQR. 4243

Results from our study demonstrate the wide range of issues that affect uptake of child restraints. As such, it is imperative for future child restraint research and programmes aimed at increasing child restraint use, focus not only on effectiveness but also embed the qualitative factors identified in our review. Implications from this review are broad. They highlight the need to develop targeted interventions for culturally and linguistically diverse groups (rather than just tailoring interventions), this may be particularly relevant in high-income countries. Further, caregivers within the same family have different perceptions (of injury risk and the value of using a child restraint) and there is a need to take that into consideration when developing programmes and messaging. Similarly, messaging should also consider the variations of perception between different parents and caregivers, this includes some parents' perception that child restraint use is a form of discipline. Differentiating between negotiable (example, sleeping late) and non-negotiable (safety) behaviour is key to promote child restraints as children grow up. As such child restraint usage programmes should focus on aspects of effective parenting too. Such perceptions could be factored into the development of effective parenting programmes to include negotiating with children. Laws appear to be universally useful in shaping perceptions as well as promoting usage. However, considering equity is important during formulation of laws. A robust and consistent law enforcement is also key to promote usage of restraints. Finally, the impracticalities reported by parents in the included studies around using the restraints (installing them in the vehicle and properly securing the child) demonstrate the need for better designs of child restraints, which could lead to both more children being restrained and fewer errors in child restraint use.

\section{Conclusion}

Many factors affect the use of car restraints for children which should be considered when planning and developing messaging and programmes to increase child restraint use. In addition, equity issues should also be targeted when planning interventions to promote uptake. There is also a need to conduct equity 
focussed qualitative research in low-income and middle-income countries in semi-urban and rural settings, involving fathers and policy makers, service providers and enforcement agencies to understand issues around usage of child restraints in those settings in the future.

\section{Competing Interests:}

$\mathrm{KH}$ authored one included study and therefore was not involved in data extraction or quality assessment. No other competing interests to declare.

\section{Funding:}

This systematic review was funded through an Oxford University Internal HEFCE GCRF grant (ref no. 005105). KH was funded through a NSW Health Early Mid-Career Research Fellowship.

\section{What is already known on this subject?}

- Child restraint usage will save children's lives in the event of a crash.

- Many countries have child restraint laws but parents still do not use them. It is not clear what the barriers and/or facilitating factors are in this regard.

\section{What this study adds}

- To our knowledge, this is the first qualitative evidence synthesis on the topic.

- It provides important insights into understanding reasons behind compliance or non-compliance of child restraint usage.

- It will inform the design of programmes locally to increase the usage of child restraints.

- It will help countries achieve Target 8 of the Global Voluntary performance Targets for Road Safety which specifically aims to increase the use of standard child restraint systems to $100 \%$ by 2030.

\section{References}

1. Foreman KJ, Marquez N, Dolgert A, et al. Forecasting life expectancy, years of life lost, and all-cause and cause-specific mortality for 250 causes of death: reference and alternative scenarios for 2016-40 for 195 countries and territories. Lancet (London, England) 2018;392(10159):2052-90. doi: 10.1016/s0140-6736(18)31694-5 [published Online First: 2018/10/21]

2. WHO. Global status report on road safety 2018. Geneva: World Health Organization, 2018.

3. Tran N. Voluntary Global Performance Targets for Road Safety Risk Factors and Service Mechanisms. In: WHO, ed. Geneva: World Health Organisation, 2018.

4. Ojo TK. Seat belt and child restraint use in a developing country metropolitan city. Accident; analysis and prevention 2018;113:325-29. doi: 10.1016/j.aap.2018.02.008 [published Online First: 2018/02/27] 
5. Lee LK, Farrell CA, Mannix R. Restraint use in motor vehicle crash fatalities in children 0 year to 9 years old. The journal of trauma and acute care surgery 2015;79(3 Suppl 1):S55-60. doi: 10.1097/ ta.0000000000000673 [published Online First: 2015/08/27]

6. Yang JZ, Li LP, Wu HQ, et al. A comparative analysis of child passenger restraint use in China and the United States. World journal of pediatrics : WJP 2017;13(6):593-98. doi: 10.1007/ s12519-017-0057-y [published Online First: 2017/07/29]

7. Fildes B, Stevenson M, Hoque $S$, et al. Restraint use in the Eastern Province of the Kingdom of Saudi Arabia. Traffic Inj Prev 2016;17(5):488-94. doi: 10.1080/15389588.2015.1103849 [published Online First: 2015/10/29]

8. Simniceanu A, Richmond SA, Snowdon A, et al. Child restraint use in Canadian provinces with and without legislation in 2010. Traffic Inj Prev 2014;15(7):734-9. doi: 10.1080/15389588.2013.867483 [published Online First: 2013/11/28]

9. Kakefuda I, Yamanaka T, Stallones L, et al. Child restraint seat use behavior and attitude among Japanese mothers. Accident; analysis and prevention 2008;40(3):1234-43. doi: 10.1016/ j.aap.2008.01.013 [published Online First: 2008/05/08]

10. Ehiri JE, Ejere HOD, Magnussen L, et al. Interventions for promoting booster seat use in four to eight year olds travelling in motor vehicles. Cochrane Database of Systematic Reviews 2006(1) doi: 10.1002/14651858.CD004334.pub2

11. Cargo M, Harris J, Pantoja T, et al. Cochrane Qualitative and Implementation Methods Group guidance series-paper 4: methods for assessing evidence on intervention implementation. Journal of clinical epidemiology 2018;97:59-69. doi: 10.1016/j.jclinepi.2017.11.028 [published Online First: 2017/12/11]

12. Flemming K, Booth A, Hannes K, et al. Cochrane Qualitative and Implementation Methods Group guidance series-paper 6: reporting guidelines for qualitative, implementation, and process evaluation evidence syntheses. Journal of clinical epidemiology 2018;97:79-85. doi: 10.1016/ j.jclinepi.2017.10.022 [published Online First: 2017/12/10]

13. Harden A, Thomas J, Cargo M, et al. Cochrane Qualitative and Implementation Methods Group guidance series-paper 5: methods for integrating qualitative and implementation evidence within intervention effectiveness reviews. Journal of clinical epidemiology 2018;97:70-78. doi: 10.1016/j.jclinepi.2017.11.029 [published Online First: 2017/12/16]

14. Harris JL, Booth A, Cargo M, et al. Cochrane Qualitative and Implementation Methods Group guidance series-paper 2: methods for question formulation, searching, and protocol development for qualitative evidence synthesis. Journal of clinical epidemiology 2018;97:39-48. doi: 10.1016/j.jclinepi.2017.10.023 [published Online First: 2017/12/19]

15. Noyes J, Booth A, Cargo M, et al. Cochrane Qualitative and Implementation Methods Group guidance series-paper 1: introduction. Journal of clinical epidemiology 2018;97:35-38. doi: 10.1016/ j.jclinepi.2017.09.025 [published Online First: 2017/12/16]

16. Noyes J, Booth A, Flemming K, et al. Cochrane Qualitative and Implementation Methods Group guidance series-paper 3: methods for assessing methodological limitations, data extraction and synthesis, and confidence in synthesized qualitative findings. Journal of clinical epidemiology 2018;97:49-58. doi: 10.1016/j.jclinepi.2017.06.020 [published Online First: 2017/12/17]

17. O'Neill J, Tabish $\mathrm{H}$, Welch $\mathrm{V}$, et al. Applying an equity lens to interventions: using PROGRESS ensures consideration of socially stratifying factors to illuminate inequities in health. Journal of clinical epidemiology 2014;67(1):56-64. doi: 10.1016/j.jclinepi.2013.08.005

18. CASP. Critical Appraisal Skills Programme Checklist Qualitative Study

2018 [Available from: https://casp-uk.net/wp-content/uploads/2018/01/CASP-QualitativeChecklist-2018.pdf accessed 25 July 2018. 
19. Thomas J, Harden A. Methods for the thematic synthesis of qualitative research in systematic reviews. BMC medical research methodology 2008;8:45. doi: 10.1186/1471-2288-8-45 [published Online First: 2008/07/12]

20. Booth A, Noyes J, Flemming K, et al. Structured methodology review identified seven (RETREAT) criteria for selecting qualitative evidence synthesis approaches. Journal of clinical epidemiology 2018;99:41-52. doi: 10.1016/j.jclinepi.2018.03.003 [published Online First: 2018/03/20]

21. Rivara FP, Bennett E, Crispin B, et al. Booster seats for child passengers: lessons for increasing their use. Injury prevention : journal of the International Society for Child and Adolescent Injury Prevention 2001;7(3):210-3. [published Online First: 2001/09/22]

22. Green L, Kreuter M. Health Program Planning: An Educational and Ecological Approach, Volumes 1-2. 4th Edition ed. New York: McGraw-Hill 2005.

23. Simpson EM, Moll EK, Kassam-Adams N, et al. Barriers to booster seat use and strategies to increase their use. Pediatrics 2002;110(4):729-36. [published Online First: 2002/10/03]

24. Lee JW, Fitzgerald K, Ebel BE. Lessons for increasing awareness and use of booster seats in a Latino community. Injury prevention : journal of the International Society for Child and Adolescent Injury Prevention 2003;9(3):268-9. [published Online First: 2003/09/11]

25. Agran PF, Anderson CL, Winn DG. Violators of a child passenger safety law. Pediatrics 2004;114(1):109-15.

26. Lennon A. A risky treat: exploring parental perceptions of the barriers to seating their children in the rear seats of passenger vehicles. Injury prevention : journal of the International Society for Child and Adolescent Injury Prevention 2007;13(2):105-9. doi: 10.1136/ip.2006.012906 [published Online First: 2007/04/21]

27. Van Manen M. Researching Lived Experience: Human Science for an Action Sensitive Pedagogy. [Albany NY]: State University of New York Press, 1990. Curriculum Inquiry 1990;24(2):135-70.

28. Medoff-Cooper B, Tulman L. Using a focus group to determine car seat use among mothers of children aged 3 to 7 years. MCN The American journal of maternal child nursing 2007;32(3):165-9. doi: 10.1097/01.NMC.0000269566.27734.e0 [published Online First: 2007/05/05]

29. Winston FK, Erkoboni D, Xie D. Identifying interventions that promote belt-positioning booster seat use for parents with low educational attainment. The Journal of trauma 2007;63(3 Suppl):S29-38. doi: 10.1097/TA.0b013e31812f5ebb [published Online First: 2007/09/14]

30. Johnston BD, Bennett E, Quan L, et al. Factors influencing booster seat use in a multiethnic community: lessons for program implementation. Health promotion practice 2009;10(3):411-8. doi: 10.1177/1524839908317743 [published Online First: 2008/06/10]

31. Erkoboni D, Ozanne-Smith J, Rouxiang C, et al. Cultural translation: acceptability and efficacy of a USbased injury prevention intervention in China. Injury prevention : journal of the International Society for Child and Adolescent Injury Prevention 2010;16(5):296-301. doi: 10.1136/ ip.2009.023341 [published Online First: 2010/10/06]

32. Brown J, Burton D, Nikolin S, et al. A qualitative approach using the integrative model of behaviour change to identify intervention strategies to increase optimal child restraint practices among culturally and linguistically diverse families in New South Wales. Injury prevention : journal of the International Society for Child and Adolescent Injury Prevention 2013;19(1):6-12. doi: 10.1136/ injuryprev-2011-040211 [published Online First: 2012/05/29]

33. Fishbein M, Cappella JN. The role of theory in developing effective health communications. Journal of communication 2006;56:S1-S17.

34. Chen X, Yang J, Peek-Asa C, et al. Parents' experience with child safety restraint in China. BMC public health 2014;14:318. doi: 10.1186/1471-2458-14-318 [published Online First: 2014/04/09]

35. Nelson A, Modeste N, Hopp Marshak H, et al. Saudi women's beliefs on the use of car infant restraints: a qualitative study. Traffic Inj Prev 2015;16:240-5. doi: 10.1080/15389588.2014.931578 [published Online First: 2014/06/10] 
36. Ajzen I. The theory of planned behavior. Organizational behavior and human decision processes 1991;50(2):179-211.

37. Liu X, Yang J, Chen X, et al. Knowledge, Attitudes and Behaviors on Child Passenger Safety among Expectant Mothers and Parents of Newborns: A Qualitative and Quantitative Approach. PloS one 2016;11(1):e0146121. doi: 10.1371/journal.pone.0146121 [published Online First: 2016/01/07]

38. Fleisher L, Erkoboni D, Halkyard K, et al. Are mHealth Interventions to Improve Child Restraint System Installation of Value? A Mixed Methods Study of Parents. International journal of environmental research and public health 2017;14(10) doi: 10.3390/ijerph14101122 [published Online First: 2017/09/29]

39. Hunter K, Keay L, Clapham K, et al. "He's the Number One Thing in My World": Application of the PRECEDE-PROCEED Model to Explore Child Car Seat Use in a Regional Community in New South Wales. International journal of environmental research and public health 2017;14(10) doi: 10.3390/ijerph14101206 [published Online First: 2017/10/11]

40. McKenzie LB, Fowler E, Roberts KJ, et al. Child Passenger Safety in the Somali Communities of Columbus, Ohio. Journal of community health 2017;42(2):221-27. doi: 10.1007/ s10900-016-0246-y [published Online First: 2016/09/11]

41. Hall A, Ho C, Keay L, et al. Barriers to correct child restraint use: A qualitative study of child restraint users and their needs. Safety Science 2018;109:186-94.

42. Tong A, Sainsbury P, Craig J. Consolidated criteria for reporting qualitative research (COREQ): a 32item checklist for interviews and focus groups. International Journal for Quality in Health Care 2007;19(6):349-57. doi: 10.1093/intqhc/mzm042

43. O'Brien BC, Harris IB, Beckman TJ, et al. Standards for reporting qualitative research: a synthesis of recommendations. Acad Med 2014;89(9):1245-51. doi: 10.1097/acm.0000000000000388 [published Online First: 2014/07/01] 